\title{
Step tolling in an activity-based bottleneck model
}

\author{
Zhi-Chun Li, ${ }^{\mathrm{a}^{*}}$ William H. K. Lam, ${ }^{\mathrm{b}, \mathrm{c}}$ S. C. Wong ${ }^{\mathrm{d}}$ \\ ${ }^{a}$ School of Management, Huazhong University of Science and Technology, Wuhan 430074, China \\ ${ }^{\mathrm{b}}$ Department of Civil and Environmental Engineering, The Hong Kong Polytechnic University, Kowloon, \\ Hong Kong, China \\ ${ }^{\mathrm{c} S}$ School of Traffic \& Transportation, Beijing Jiaotong University, Beijing 100044, China \\ ${ }^{\mathrm{d}}$ Department of Civil Engineering, The University of Hong Kong, Pokfulam Road, Hong Kong, China
}

\begin{abstract}
This paper investigates the step tolling problem in an activity-based bottleneck model in which activity scheduling utilities of commuters at home and at work vary by the time of day. The commuters choose their departure times from home to work in the morning to maximize their own scheduling utility. Step tolling models with homogeneous and heterogeneous preferences are presented. The properties of the models and the optimal step toll schemes with constant and linear time-varying marginal activity utilities are analytically explored and compared. It was found that for a given number of toll steps the efficacy of a step toll in terms of queuing removal rate is higher in the activity-based bottleneck model with linear marginal utilities than in the conventional bottleneck model with constant marginal utilities, and ignoring the preference heterogeneity of commuters would underestimate the efficacy of a step toll.
\end{abstract}

Keywords: step tolling; activity-based bottleneck model; homogeneous and heterogeneous preferences; time-varying marginal activity utility.

\footnotetext{
${ }^{*}$ Corresponding author. Tel.: +86-27-8755-6490; fax: +86-27-8755-6437.

E-mail address: smzcli@gmail.com (Z.-C. Li).
} 


\section{Introduction}

The bottleneck model introduced by Vickrey (1969) has been widely recognized as a useful tool for modeling the formation and dissipation of queuing at a bottleneck during the morning peak (Small, 2015). A queuing delay is a pure deadweight loss for drivers and results in inefficient use of the transportation infrastructure. It has been shown that a triangular and time-varying toll scheme can be used in the bottleneck model to totally eliminate the annoying queue behind the bottleneck to achieve the full social optimum. However, a continuously time-varying toll scheme can hardly be implemented in reality because a continuously changing toll structure may confuse drivers due to cognitive barrier or bounded rationality so that they may not be able to respond effectively to the price signals. Step toll (single-step or multistep) schemes have thus been adopted in practice as an approximation and substitute for the time-varying toll scheme.

For instance, the London congestion toll pricing system has a uniform (or single-step) toll of $£ 11.50$ from 07:00 to 18:00 on weekdays. ${ }^{1}$ Singapore's Electronic Road Pricing (ERP) system uses a multistep toll scheme (the Land Transport Authority (LTA) of Singapore calls it "shoulder pricing"), similar to those of Stockholm, the SR91 and the Bay Bridge in California, and the SR520 and SR16 bridges in the state of Washington. The step tolls for passenger cars on arterial roads in Singapore are between S\$0.5 and S\$3.0 ("S\$" stands for Singapore's currency; S\$1 was approximately US\$0.70 on 1 Jan 2017). ${ }^{2}$ Unlike the first-best toll scheme that can completely remove the queue at the bottleneck, the step tolling schemes can remove part of queue only. This raises an interesting and important issue: how to design an efficient step tolling scheme to maximize the scheduling utility of commuters, particularly when the commuters are divided into different classes according to their marginal work utility (or income level per unit of time).

In the literature, the studies on step tolling in the bottleneck model can be classified into three categories: the ADL model of Arnott et al. (1990, 1998), the Laih model of Laih (1994, 2004), and the braking model of Lindsey et al. (2012) and Xiao et al. (2012). The Laih model implicitly assumes that separate queues exist for tolled users and untolled users who arrive

\footnotetext{
${ }^{1}$ https://tfl.gov.uk/modes/driving/congestion-charge. Retrieved on 1 Jan 2017.

${ }^{2}$ http://www.onemotoring.com.sg/publish/onemotoring/en/on_the_roads/ERP_Rates.html. Retrieved on 1 Jan 2017.
} 
after the toll is turned off. Despite this strong assumption, the Laih model is useful for estimating the approximate efficiency of a multistep toll scheme. The ADL model assumes that a mass of commuters departs just after the toll is lifted. The braking model considers that as the end of the tolling period approaches, drivers have an incentive to stop before reaching the tolling point and wait until the toll is switched off. For comprehensive reviews of the step tolling models, readers can refer to Lindsey et al. (2012) and van den Berg (2012, 2014). The Laih method is adopted in this paper because it provides a simple way to derive the optimal multistep toll scheme.

The studies on step tolling are usually based on Vickrey's bottleneck model, in which individuals have a preferred time to arrive at their destination and incur a schedule delay cost proportional to the amount of time that they arrive early or late. Vickrey's bottleneck model treats the trip scheduling problem for the morning commute by modeling the trade-off between bottleneck congestion and schedule delay. It assumes that the value of travel time and the value of schedule delay are constants, i.e., the marginal cost of travel time and the marginal costs of arriving early and late at work are, respectively, assumed to be constants $\alpha$, $\beta$, and $\gamma$. Such scheduling preferences are referred to as $\alpha-\beta-\gamma$ preferences in Knockaert et al. (2016). The assumption of the $\alpha-\beta-\gamma$ preferences has been widely adopted in various extensions or variations of the traditional Vickrey's bottleneck model, such as the ADL model, the Laih model and the braking model. It will be shown in the later section of this paper that the Vickrey's bottleneck model with $\alpha-\beta-\gamma$ preferences is actually an activity-based model with constant marginal activity utilities.

However, some empirical studies have confirmed that the marginal utility of time for performing an activity at a certain location varies over time (see, e.g., Ettema and Timmermans, 1997, 2003; Liu et al., 2007; Tseng and Verhoef, 2008; Jenelius et al., 2011). In this regard, Vickrey (1973) formulated the departure time choice model for the morning commuting problem (also called the scheduling model of morning commute), in which the utilities derived from time spent at home and at work are linear functions of time. Fosgerau and Engelson (2011) used the linear time-varying marginal activity utilities to investigate the value of travel time variability. Börjesson et al. (2012) further compared the valuations of travel time variability with the mean-variance model and the scheduling model with linear time-varying marginal activity utilities. Tseng and Verhoef (2008) calibrated the scheduling 
model of the morning commute, in which marginal utilities are allowed to vary nonlinearly over time. Fosgerau and de Palma (2012) presented a model with more general scheduling preferences to analyze commuting behavior in a city where workers live at various distances from the CBD. Fosgerau and Lindsey (2013) further analyzed traffic bottleneck congestion and commuters' trip-timing decisions when drivers randomly cause incidents that temporarily block the bottleneck. However, the properties of the scheduling model with the time-varying marginal activity utilities and its applications in the step tolling issues have received surprisingly little attention in the literature.

In addition, these studies accounting for time-varying marginal activity utilities usually assumed that all commuters were homogeneous (i.e., only one user class), and thus the effects of the preference heterogeneity of commuters (i.e., multiple user classes) were not considered. However, studies have found that heterogeneity in trip-timing preferences is significant (see e.g., Small et al., 2005) and heterogeneous commuters exhibit a large behavioral difference in departure time choice during peak hours (see, e.g., Arnott et al., 1988; Lindsey, 2004; Wu and Huang, 2015) and in the response to the congestion tolls (Cohen, 1987; Arnott et al., 1994; Huang, 2000; Xiao et al., 2011; van den Berg and Verhoef, 2011a,b; Yao et al., 2012; van den Berg, 2014; Wu and Huang, 2014; Chen et al., 2015). Ignoring the preference heterogeneity may cause a biased estimation of the efficacy of congestion tolls. It is, thus, of great importance to incorporate the preference heterogeneity of commuters in the congestion tolling problems. However, to the best of our knowledge, the existing congestion tolling studies considering the preference heterogeneity basically fall into the family of the $\alpha-\beta-\gamma$ preferences (i.e., a case with constant marginal activity utilities), and little attention has been paid to the case with time-varying marginal activity utilities.

In light of the above, this paper studies the step tolling problem in the activity-based bottleneck model with time-varying marginal activity utilities. Two types of scheduling preferences, namely homogeneous and heterogeneous preferences, are considered. This paper makes three main contributions to the previous related studies. First, the closed-form solution of the activity-based bottleneck model with time-varying marginal activity utilities is derived, and its properties are analytically explored, particularly for instances in which the marginal activity utilities are a linear function of time. The linear marginal activity utilities can be considered as an approximation of any marginal activity utility functions through the first-order Taylor expansion. Therefore, investigation on the linear marginal activity utility 
case may provide an avenue for evaluating the solution of the model with general scheduling preferences. Second, single-step and multistep toll schemes with linear time-varying marginal activity utilities are analytically investigated and compared with those with constant marginal activity utilities (i.e., traditional bottleneck model with $\alpha-\beta-\gamma$ preferences). The results show that the optimal step toll scheme with linear marginal activity utilities follows a symmetric (or shoulder) toll structure. For a given number of toll steps, the efficacy of a step toll in terms of queuing removal rate is higher in the activity-based bottleneck model with linear marginal activity utilities than in the conventional bottleneck model with constant marginal activity utilities. Third, the heterogeneity of user preferences is incorporated in the activity-based bottleneck model and the step tolling problem. It is found that heterogeneous groups sequentially depart in order of decreasing marginal work utility or increasing marginal home activity utility, and ignoring the preference heterogeneity of commuters can cause an underestimation of the efficacy of a step toll.

The remainder of this paper is organized as follows. In the next section, the traditional bottleneck model and the associated step tolling issue are reviewed. Section 3 presents the activity-based bottleneck model with homogeneous preferences and its properties, particularly for the linear time-varying marginal activity utility case. In Section 4, the step tolling issue with homogeneous preferences is analytically explored. Section 5 extends it to consider the preference heterogeneity of commuters. Finally, Section 6 provides the conclusions and recommendations for further study.

\section{A review of the bottleneck model}

For completeness and comparison purposes, in this section we provide a summary introduction to traditional Vickrey's bottleneck model and discuss the step tolling issue in such a classical bottleneck model. The former is mainly taken from Arnott et al. (1990, 1998), and the latter is taken from Laih $(1994,2004)$.

Vickrey's bottleneck model, as a stylized representation of the dynamics of traffic congestion, addresses the departure time choice problem of commuters during the morning peak. Suppose that every morning, $N$ commuters travel from home to a workplace along a single road that has a bottleneck with a fixed capacity s. All of these commuters wish to arrive at their 
workplace at an identical preferred arrival time $t^{*}$. Without loss of generality, the free-flow travel time from home to work is assumed to be zero. Thus, a commuter arrives at the bottleneck immediately after leaving home and arrives at his or her workplace immediately after leaving the bottleneck. These assumptions do not affect the results of interest and will also be applied to the activity-based bottleneck model presented later. When the arrival rate at the bottleneck exceeds the bottleneck's capacity, a queue develops. Those who arrive early or late encounter a schedule delay cost. Commuters choose their departure times on the basis of a trade-off between the bottleneck congestion and the schedule delay cost.

Let $C(t)$ represent the travel cost of commuters departing from home to work at time $t$. It consists of the travel time cost (i.e., the queuing delay cost at the bottleneck) and the schedule delay cost of arriving early or late. Let $T(t)$ represent the travel time (or queuing delay time) at the bottleneck at time $t$. $C(t)$ can then be expressed as

$$
C(t)=\alpha T(t)+\beta \max \left(0, t^{*}-t-T(t)\right)+\gamma \max \left(0, t+T(t)-t^{*}\right),
$$

where $\alpha$ is the unit cost of travel time, $\beta$ is the unit cost of arriving early, and $\gamma$ is the unit cost of arriving late. Following the empirical study of Small (1982), the relationship $\gamma>\alpha>\beta$ should hold.

The queuing delay time $T(t)$ at the bottleneck at time $t$ equals the queue length $D(t)$ divided by the bottleneck capacity $s$, i.e., $T(t)=D(t) / s$, where $D(t)$ equals the difference between the cumulative arrivals and cumulative departures by that time, i.e.,

$$
D(t)=\int_{t_{q}}^{t} r(t) d t-s\left(t-t_{q}\right)
$$

where $r(t)$ is the departure rate of commuters from home at time $t$ and $t_{q}$ is the time at which the queue begins.

At the equilibrium, all commuters experience the same travel cost $C(t)$ in the morning peak period regardless of their departure times. This means $d C(t) / d t=0, \forall t \in\left(t_{q}, t_{q^{\prime}}\right)$, where $t_{q^{\prime}}$ is the time when the queue ends and $\left(t_{q}, t_{q^{\prime}}\right)$ is the morning peak period. Hence, one can easily derive the equilibrium departure rate $r(t)$ as 


$$
r(t)= \begin{cases}\frac{\alpha}{\alpha-\beta} s, & \forall t \in\left(t_{q}, \tilde{t}\right), \\ \frac{\alpha}{\alpha+\gamma} s, & \forall t \in\left(\tilde{t}, t_{q^{\prime}}\right),\end{cases}
$$

where $\tilde{t}$ denotes the departure time from home at which a commuter can arrive at workplace on time, i.e., $\tilde{t}+T(\tilde{t})=t^{*}$. For the purposes of illustration, Fig. 1a depicts the equilibrium departure rate of commuters in the morning peak period, which is piecewise constant. Fig. $1 \mathrm{~b}$ plots the cumulative departures from home and the cumulative arrivals at workplace. The cumulative departure curve is piecewise linear.

In the morning peak $\left(t_{q}, t_{q^{\prime}}\right)$, the capacity of the bottleneck is reached, and thus $t_{q^{\prime}}-t_{q}=N / s$ holds. At the equilibrium, the first and last commuters do not face a queue, their queuing delays are zero, and their schedule delay costs must thus be equal, expressed as

$$
\beta\left(t^{*}-t_{q}\right)=\gamma\left(t_{q^{\prime}}-t^{*}\right) \text {. }
$$

From Eq. (4) together with $t_{q^{\prime}}-t_{q}=N / s$ and $\tilde{t}+T(\tilde{t})=t^{*}$, one obtains

$$
t_{q}=t^{*}-\frac{\gamma}{\beta+\gamma} \frac{N}{s}, t_{q^{\prime}}=t^{*}+\frac{\beta}{\beta+\gamma} \frac{N}{s} \text {, and } \tilde{t}=t^{*}-\frac{\beta \gamma}{\alpha(\beta+\gamma)} \frac{N}{s} .
$$

The resultant equilibrium travel cost $\bar{C}$ is

$$
\bar{C}=\frac{\beta \gamma}{\beta+\gamma} \frac{N}{S} .
$$

From equilibrium condition $C(t)=C\left(t_{q}\right)=C\left(t_{q^{\prime}}\right)$ and Eqs. (1) and (4), we can derive the queuing delay time as

$$
T(t)= \begin{cases}\frac{\beta}{\alpha-\beta}\left(t-t_{q}\right), \quad \forall t \in\left[t_{q}, \tilde{t}\right], \\ \frac{\gamma}{\alpha+\gamma}\left(t_{q^{\prime}}-t\right), \quad \forall t \in\left[\tilde{t}, t_{q^{\prime}}\right] .\end{cases}
$$

Eq. (7) shows that a queue builds up linearly from $t_{q}$ to $\tilde{t}$ and then dissipates linearly until it disappears at $t_{q^{\prime}}$. Fig. 1c depicts the change of the queuing delay time $T(t)$ during the morning peak $\left(t_{q}, t_{q^{\prime}}\right)$. It can be noted that the queuing delay curve (also referred to as isocost 
queuing curve in Cohen (1987)) is piecewise linear.

A queuing delay is a pure deadweight loss in the bottleneck model. At the social optimum (i.e., the first-best) situation, queuing at the bottleneck should be eliminated and the capacity of the bottleneck should be fully utilized. To do so, the optimal (first-best) time-varying tolls can be imposed to replace the queuing delay such that the no-toll user equilibrium can automatically be driven to the social optimum. The social optimum time-varying congestion tolls are presented as follows.

$$
\tau(t)= \begin{cases}\frac{\beta \gamma}{\beta+\gamma} \frac{N}{s}-\beta\left(t^{*}-t\right), & t \in\left[t_{q}, t^{*}\right], \\ \frac{\beta \gamma}{\beta+\gamma} \frac{N}{s}-\gamma\left(t-t^{*}\right), & t \in\left[t^{*}, t_{q^{\prime}}\right],\end{cases}
$$

where $\tau(t)$ denotes the congestion toll at the bottleneck at time $t$.

Remark 1. According to Eqs. (7) and (8), one can easily derive the following relationship:

$$
\frac{\tau(t)}{T(t)}= \begin{cases}\alpha-\beta, & t \in\left(t_{q}, t^{*}\right), \\ \alpha+\gamma, & t \in\left(t^{*}, t_{q^{\prime}}\right) .\end{cases}
$$

This means that decreasing one unit of queuing time at the bottleneck requires increasing the tolls by $(\alpha-\beta)$ units for early arrival period, and $(\alpha+\gamma)$ units for late arrival period.

As previously stated, cognitive barrier or bounded rationality of commuters makes the continuously time-varying toll scheme difficult to implement in reality. Therefore, step tolling (single-step or multistep) schemes are usually adopted in practice. Laih (1994) indicated the properties of the step toll schemes in Vickrey's bottleneck model as follows.

Lemma 1. The optimal $m$-step toll scheme divides the maximum optimal time-varying toll into $(m+1)$ equal amounts and eliminates at most $m /(m+1)$ of the total queuing delay that exists under the no-toll equilibrium.

For the purposes of illustration, Figs. $2 \mathrm{a}$ and $2 \mathrm{~b}$ indicate the optimal single-step and two-step toll systems for the Laih model. It can be noted that the single-step toll is half the maximum optimal time-varying toll $\tau_{\max }=\frac{\beta \gamma}{\beta+\gamma} \frac{N}{S}$, causing a removal of half the total queuing delay 
under the no-toll equilibrium. The two-step tolls are one third and two thirds of the maximum optimal time-varying toll $\tau_{\max }$, respectively. As a result, two thirds of the total queuing delay under the no-toll equilibrium is removed. For more details, readers can refer to Laih (1994).

\section{Activity-based bottleneck model with homogeneous preferences}

In this section, we first present a general formulation of the activity-based bottleneck model with homogeneous preferences and its properties. The preference homogeneity means that all commuters in the system have the same marginal activity utility functions and parameter values (i.e., only one user class). Two special cases of the model are then discussed: constant marginal activity utility case and linear marginal activity utility case.

\subsection{A general formulation}

We consider a morning commuting schedule that consists of two activities (i.e., being at home and being at work) and one trip (i.e., the journey from home to work), as shown in Fig. 3. Every morning, $N$ commuters travel from home to a workplace connected by a single road that is subject to bottleneck queuing congestion. Commuters can gain utility from being at home and from being at work. We define a clock time $t_{s}$ as the morning start time and a clock time $t_{E}$ as the morning end time. Both are arbitrarily chosen such that all travel takes place within the interval $\left[t_{S}, t_{E}\right]$. For example, $t_{S}$ is chosen early enough and $t_{E}$ is chosen late enough. We denote $t_{d}$ as the departure time of a commuter from home and $t_{a}$ as the arrival time of a commuter at workplace. The free-flow travel time from home to work is normalized to be zero, as in the traditional Vickrey's bottleneck model. A commuter departing at $t_{d}$ encounters a queuing time of $T\left(t_{d}\right)$ at the bottleneck and reaches the workplace at time $t_{a}=t_{d}+T\left(t_{d}\right)$.

The departure time choices of commuters during the morning peak are based on the trade-off between the utilities of activities performed at home and at work and the journey time from home to work. Commuters choose their departure times from home to work in the morning interval $\left[t_{S}, t_{E}\right]$ to maximize their own scheduling utility. To represent commuters' scheduling utility, we define the marginal utilities of being at home, at work, and in a vehicle 
as follows. Let $h(t)$ represent the marginal utility of being at home relative to the marginal utility of being in a vehicle at time $t$, and let $w(t)$ represent the marginal utility of being at work relative to the marginal utility of being in a vehicle at time $t$. Without loss of generality, the utility of being in a vehicle is normalized to zero. Thus, for given departure time $t_{d}$ and arrival time $t_{a}$, the total utility derived from activities in the morning interval $\left[t_{s}, t_{E}\right]$ can be represented as

$$
U\left(t_{d}, t_{a}\right)=\int_{t_{S}}^{t_{d}} h(t) d t+\int_{t_{a}}^{t_{E}} w(t) d t .
$$

The formulation of such scheduling utilities originates from Vickrey (1973) and has been applied by Tseng and Verhoef (2008), Engelson and Fosgerau (2011), Fosgerau and Engelson (2011), Jenelius et al. (2011), Börjesson et al. (2012), Fosgerau and de Palma (2012), Fosgerau and Lindsey (2013), and Hjorth et al. (2015). However, these previous related studies did not carry out an in-depth analysis of the properties of the scheduling utility function, which is presented as follows.

The scheduling utility defined in Eq. (10) depends on the chosen times $t_{S}$ and $t_{E}$. For the purposes of presentation, we normalize the scheduling utility function to eliminate the effects of $t_{S}$ and $t_{E}$. In this paper, both $h(t)$ and $w(t)$ are assumed to be positive to guarantee that the value of travel time saving is positive. To motivate making a trip, at some time point the value of $w(t)$ must exceed the value of $h(t)$. We assume that $h(t)$ monotonically decreases and that $w(t)$ monotonically increases and that a time $t^{* *}$ exists such that $h(t)>w(t)$ for $t<t^{* *}$ and $h(t)<w(t)$ for $t>t^{* *}$. These assumptions have been justified in previous empirical studies (see, e.g., Tseng and Verhoef, 2008; Börjesson et al., 2012), and have also been adopted in the studies of Engelson and Fosgerau (2011), Fosgerau and Lindsey (2013), and Hjorth et al. (2015). Under these assumptions, travelers prefer to be at home before $t^{* *}$ and at work after $t^{* *}$. Referring to Fig. 4, the potential maximum scheduling utility that one commuter can achieve in the morning interval $\left[t_{S}, t_{E}\right]$ is

$$
U\left(t^{* *}, t^{* *}\right)=\int_{t_{S}}^{t^{* *}} h(t) d t+\int_{t^{* *}}^{t_{E}} w(t) d t .
$$

We now define the relative scheduling utility of commuters as the difference between $U\left(t_{d}, t_{a}\right)$ and $U\left(t^{* *}, t^{* *}\right)$, that is, 


$$
\begin{aligned}
\hat{U}\left(t_{d}, t_{a}\right) & =U\left(t_{d}, t_{a}\right)-U\left(t^{* *}, t^{* *}\right) \\
& =-\int_{t_{d}}^{t^{* *}} h(t) d t-\int_{t^{* *}}^{t_{a}} w(t) d t .
\end{aligned}
$$

Accordingly, the scheduling utility of commuters in the morning can be evaluated at $t^{* *}$ rather than at $t_{S}$ and $t_{E}$ in terms of Eq. (12).

For the purpose of presentation, we define the scheduling opportunity cost (also referred to as travel cost in this paper) of the morning commute starting at $t_{d}$ and ending at $t_{a}$ as

$$
C\left(t_{d}, t_{a}\right)=\int_{t_{d}}^{t^{* *}} h(t) d t+\int_{t^{* *}}^{t_{a}} w(t) d t
$$

or equivalently

$$
C\left(t_{d}\right)=\int_{t_{d}}^{t^{* *}} h(t) d t+\int_{t^{* *}}^{t_{d}+T\left(t_{d}\right)} w(t) d t .
$$

Hence, maximizing the scheduling utility $U\left(t_{d}, t_{a}\right)$ in Eq. (10) for the choice of departure time $t_{d}$ in the morning is equivalent to minimizing the travel cost $C\left(t_{d}, t_{a}\right)$ or $C\left(t_{d}\right)$ in Eq. (13) or (14). The scheduling model (or called activity-based bottleneck model in this paper) for the morning commute can thus mathematically be formulated as

$$
\min _{t_{d}} C\left(t_{d}, t_{a}\right) \text { or } \min _{t_{d}} C\left(t_{d}\right) .
$$

Fig. 4 is used to graphically illustrate the implications of Eqs. (10)-(14). Note that there are three possible relationships among $t_{d}, t_{a}$, and $t^{* *}$ : (i) $t_{d}$ is before $t^{* *}$ and $t_{a}$ is after $t^{* *}$ (see Fig. 4a); (ii) both $t_{d}$ and $t_{a}$ are before $t^{* *}$ (see Fig. 4b); and (iii) both $t_{d}$ and $t_{a}$ are after $t^{* *}$ (see Fig. 4c). The scheduling utility of performing home or work activity over a period can be graphically identified as the area of the region below the marginal utility curve $h(t)$ or $w(t)$ over that period, as indicated in Fig. 4.

The scheduling utilities and costs for these three cases are, respectively, given as follows. The scheduling utility $U\left(t_{d}, t_{a}\right)$ of commuters over the morning interval $\left[t_{s}, t_{E}\right]$ is the sum of $\int_{t_{S}}^{t_{d}} h(t) d t$ and $\int_{t_{a}}^{t_{E}} w(t) d t$ in terms of Eq. (10). For case (i), the former is the sum of the areas of $I$ and $I I$, whereas the latter is the sum of the areas of VII and VIII. We thus have $U\left(t_{d}, t_{a}\right)=(I+I I)+(V I I+V I I I)$, which are the areas marked in red in Fig. 4a. Note that the 
potential maximum scheduling utility $U\left(t^{* *}, t^{* *}\right)$ is the sum of the areas of all of the partitions below the marginal utility curves $h(t)$ and $w(t)$, i.e., $U\left(t^{* *}, t^{* *}\right)=I+I I+I I I+I V+V+V I+V I I+V I I I$. The scheduling opportunity cost $C\left(t_{d}, t_{a}\right)$, which is the difference between $U\left(t^{* *}, t^{* *}\right)$ and $U\left(t_{d}, t_{a}\right)$, can thus be represented as

$$
C\left(t_{d}, t_{a}\right)=U\left(t^{* *}, t^{* *}\right)-U\left(t_{d}, t_{a}\right)=I I I+I V+V+V I, \text { for } t_{d} \leq t^{* *} \leq t_{a},
$$

which are the areas marked in blue in Fig. 4a.

Similarly, for case (ii), $U\left(t_{d}, t_{a}\right)=(I+I I)+(V+V I+V I I)$, i.e., the areas marked in red in Fig. 4b. We thus obtain the scheduling opportunity cost $C\left(t_{d}, t_{a}\right)$ as below.

$$
C\left(t_{d}, t_{a}\right)=U\left(t^{* *}, t^{* *}\right)-U\left(t_{d}, t_{a}\right)=I I I+I V, \text { for } t_{d} \leq t_{a} \leq t^{* *},
$$

which are the areas marked in blue in Fig. $4 \mathrm{~b}$.

For case (iii), $U\left(t_{d}, t_{a}\right)=(I+I I+I V)+(V I+V I I)$, i.e., the areas marked in red in Fig. 4c. One thus obtains the scheduling opportunity cost $C\left(t_{d}, t_{a}\right)$

$$
C\left(t_{d}, t_{a}\right)=U\left(t^{* *}, t^{* *}\right)-U\left(t_{d}, t_{a}\right)=I I I+V, \text { for } t^{* *} \leq t_{d} \leq t_{a},
$$

which are the areas marked in blue in Fig. 4c.

In comparison with the previous related studies, the above graphical illustration provides a clearer explanation on the implication of the travel cost function $C\left(t_{d}, t_{a}\right)$ or $C\left(t_{d}\right)$ in the activity-based bottleneck model (15). The following proposition further presents the properties of the activity-based bottleneck model (15), which are not investigated in the previous related studies. Its proof is provided in Appendix A.

Proposition 1. (i) If $h(t)$ monotonically decreases, $w(t)$ monotonically increases, and both intersect at one point, then a queue must exist at the bottleneck. (ii) The queuing delay time curve is concave.

Remark 2. Proposition 1 reveals the concavity of the queuing delay time curve. This means that the queue length at the bottleneck first increases from zero since the time $t_{q}$ that the first commuter departs from home, and then decreases to zero at the time $t_{q^{\prime}}$ that the last 
commuter departs from home. Thereby, a queue always exists for any time $t \in\left(t_{q}, t_{q^{\prime}}\right)$.

According to Eq. (2) and $T(t)=D(t) / s$, one can obtain $\frac{d T(t)}{d t}=\frac{1}{s} \frac{d D(t)}{d t}=\frac{r(t)}{s}-1$. From the first-order optimality condition of the activity-based bottleneck model (15), one obtains $\frac{d T(t)}{d t}=\frac{h(t)}{w(t+T(t))}-1 \quad$ (cf. the proof of part (ii) of Proposition 1). We thus have

Proposition 2. The equilibrium departure rate $r(t)$ of the activity-based bottleneck model (15) is

$$
r(t)=\frac{h(t)}{w(t+T(t))} s .
$$

Proposition 2 shows that the equilibrium departure rate $r(t)$ is governed by the ratio of the marginal home activity utility at departure time $t$ from home to the marginal work activity utility at arrival time $t+T(t)$ at the workplace.

\subsection{Constant marginal activity utility case}

In this subsection, we discuss the special case of constant marginal activity utilities. Suppose that the commuters' marginal utility $h(t)$ of being at home is a constant over the period concerned and that the marginal utility $w(t)$ of being at work is piecewise constant (or a step function) with a discrete jump at time $t^{* *}$ (as shown in Fig. 5a), that is,

$$
\begin{aligned}
h(t) & =\alpha . \\
w(t) & =\left\{\begin{array}{l}
\alpha-\beta, \text { if } t+T(t) \leq t^{* *}, \\
\alpha+\gamma, \text { if } t+T(t)>t^{* *}
\end{array}\right.
\end{aligned}
$$

Substituting Eqs. (20) and (21) into Eq. (13) or (14) yields the travel cost function in traditional Vickrey's bottleneck model (see Eq. (1)). This means that the traditional Vickrey's bottleneck model can be formulated as an activity-based bottleneck model with constant marginal activity utilities and is thus a special case of the activity-based bottleneck model (15). 


\subsection{Linear marginal activity utility case}

We now consider the linear marginal activity utility case. As previously stated, the linear marginal activity utilities can be regarded as an approximation of any nonlinear marginal activity utility functions through the first-order Taylor expansion. This study thus provides an avenue for roughly evaluating the solutions with general scheduling preferences.

Define $h(t)$ and $w(t)$ as the linear or affine functions of time $t$, as follows.

$$
\begin{aligned}
& h(t)=\xi+\delta t, \\
& w(t)=\mu+\lambda t,
\end{aligned}
$$

where the parameters $\xi, \mu$, and $\lambda$ are positive, and the parameter $\delta$ is negative. As stated before, in order to motivate making a trip, $w(t)$ and $h(t)$ must intersect at a time point of $t^{* *}=\frac{\xi-\mu}{\lambda-\delta}$, as depicted in Fig. 5b. Thus, $\xi>\mu$ should be satisfied if $t^{* *}>0$.

Substituting Eqs. (22) and (23) and $t^{* *}=\frac{\xi-\mu}{\lambda-\delta}$ into Eq. (14), one obtains the travel cost function $C(t)$ as below

$$
\begin{aligned}
C(t) & =\int_{t}^{t^{* *}} h(t) d t+\int_{t^{* *}}^{t+T(t)} w(t) d t \\
& =\frac{\delta}{2}\left(\left(t^{* *}\right)^{2}-t^{2}\right)-(\xi-\mu)\left(t-t^{* *}\right)+\mu T(t)+\frac{\lambda}{2}\left((t+T(t))^{2}-\left(t^{* *}\right)^{2}\right), \\
& =-\frac{\delta}{2} t^{2}-(\xi-\mu) t+\mu T(t)+\frac{\lambda}{2}(t+T(t))^{2}+\frac{(\xi-\mu)^{2}}{2(\lambda-\delta)} .
\end{aligned}
$$

The first commuter who departs from home at $t_{q}$ faces no queue due to his or her early departure from home. His or her travel cost is represented as

$$
\begin{aligned}
C\left(t_{q}\right) & =\int_{t_{q}}^{t^{* * *}}(h(t)-w(t)) d t=\frac{1}{2}(\lambda-\delta)\left(t_{q}^{2}-\left(t^{* *}\right)^{2}\right)-(\xi-\mu)\left(t_{q}-t^{* *}\right) \\
& =\frac{1}{2}(\lambda-\delta) t_{q}^{2}-(\xi-\mu) t_{q}+\frac{(\xi-\mu)^{2}}{2(\lambda-\delta)} .
\end{aligned}
$$

Similarly, the last commuter who departs at $t_{q^{\prime}}$ also faces no queue due to his or her late 
departure from home. The associated travel cost is

$$
\begin{aligned}
C\left(t_{q^{\prime}}\right) & =\int_{t^{* *}}^{t_{q^{\prime}}}(w(t)-h(t)) d t=\frac{1}{2}(\lambda-\delta)\left(t_{q^{\prime}}^{2}-\left(t^{* *}\right)^{2}\right)-(\xi-\mu)\left(t_{q^{\prime}}-t^{* *}\right) \\
& =\frac{1}{2}(\lambda-\delta) t_{q^{\prime}}^{2}-(\xi-\mu) t_{q^{\prime}}+\frac{(\xi-\mu)^{2}}{2(\lambda-\delta)} .
\end{aligned}
$$

From the equilibrium condition $C\left(t_{q}\right)=C\left(t_{q^{\prime}}\right)$, we obtain

$$
t_{q^{\prime}}+t_{q}=\frac{2(\xi-\mu)}{\lambda-\delta}
$$

According to Proposition 1 and Remark 2, for the activity-based bottleneck model with linear marginal activity utilities, a queue always exists at the bottleneck for any time $t \in\left(t_{q}, t_{q^{\prime}}\right)$. The departure rate from the bottleneck for any time $t \in\left(t_{q}, t_{q^{\prime}}\right)$ thus equals the capacity $s$ of that bottleneck. Therefore, the length of the morning peak period at the bottleneck can be calculated by

$$
t_{q^{\prime}}-t_{q}=\frac{N}{S}
$$

From Eqs. (27) and (28), we obtain

$$
\left\{\begin{array}{l}
t_{q}=\frac{\xi-\mu}{\lambda-\delta}-\frac{N}{2 s}, \\
t_{q^{\prime}}=\frac{\xi-\mu}{\lambda-\delta}+\frac{N}{2 s} .
\end{array}\right.
$$

Substituting Eq. (29) into Eqs. (25) and (26) yields the following equilibrium travel cost

$$
C(t)=C\left(t_{q}\right)=C\left(t_{q^{\prime}}\right)=\frac{N^{2}}{8 s^{2}}(\lambda-\delta) .
$$

From Eqs. (24) and (30), one can derive the equilibrium queuing delay time $T(t)$ and the time with maximum queuing delay for the activity-based bottleneck model with linear marginal activity utilities as follows.

Proposition 3. (i) The equilibrium queuing delay time $T(t)$ at the bottleneck is a quadratic (or parabolic) curve with regard to $t$, expressed as 


$$
\frac{\lambda}{2}(T(t))^{2}+(\lambda t+\mu) T(t)+\frac{\lambda-\delta}{2} t^{2}-(\xi-\mu) t+\frac{(\xi-\mu)^{2}}{2(\lambda-\delta)}-\frac{N^{2}}{8 s^{2}}(\lambda-\delta)=0, \quad t \in\left[t_{q}, t_{q^{\prime}}\right],
$$

or equivalently,

$$
T(t)=-\left(t+\frac{\mu}{\lambda}\right)+\frac{1}{\lambda} \sqrt{(\lambda t+\mu)^{2}-2 \lambda\left(\frac{\lambda-\delta}{2} t^{2}-(\xi-\mu) t+\frac{(\xi-\mu)^{2}}{2(\lambda-\delta)}-\frac{N^{2}}{8 s^{2}}(\lambda-\delta)\right)}, t \in\left[t_{q}, t_{q^{\prime}}\right] .
$$

(ii) The time with maximum queuing delay occurs at

$$
\hat{t}=-\frac{\xi}{\delta}+\frac{1}{\delta} \sqrt{\xi^{2}-\frac{\delta \lambda N^{2}}{4 s^{2}}+\frac{\delta(\xi-\mu)(2 \xi \lambda-\delta \mu-\xi \delta)}{(\lambda-\delta)^{2}}} .
$$

Remark 3. (i) $T(t)$ in Eq. (32) is continuous, differentiable, and nonlinear with regard to time $t$ under the assumption of linear marginal activity utility functions. This is an important difference from the traditional Vickrey's bottleneck model with constant marginal utilities, in which the queuing delay time curve is a piecewise linear function of time (see Eq. (7) and Fig. 1c) and is thus non-differentiable due to the assumption of constant marginal activity utility functions (see Eqs. (20) and (21)).

(ii) It can easily be shown that the second-order derivative of $T(t)$ with regard to $t$ is negative, i.e., $\frac{d^{2} T(t)}{d t^{2}}<0$. Consequently, $T(t)$ is a concave function of time $t$. This result is consistent with part (ii) of Proposition 1.

(iii) The time, $\hat{t}$, with the maximum queuing delay can directly be derived by setting $\frac{d T(\hat{t})}{d t}=0$. It is a critical time for identifying the relationship between the equilibrium departure rate $r(t)$ and the bottleneck capacity s, i.e.,

$$
r(t)\left\{\begin{array}{l}
>s, t<\hat{t}, \\
=s, t=\hat{t}, \\
<s, t>\hat{t},
\end{array}\right.
$$

where the equilibrium departure rate $r(t)$ can be given by Eq. (19).

\subsection{An illustrative example}

In this section, a numerical example is employed to illustrate the properties of the proposed 
activity-based bottleneck model with linear marginal activity utilities. The marginal utility functions for home and work activities are assumed to follow Eqs. (22) and (23). The empirical results for parameters $\delta$ and $\lambda$ of Tseng and Verhoef (2008) are adopted: $\delta=-8.86\left(\$ / \mathrm{h}^{2}\right)$ and $\lambda=25.42\left(\$ / \mathrm{h}^{2}\right)$. $\xi$ and $\mu$ are assumed to $\$ 57$ and $\$ 40$ per hour, respectively. The total number of commuters $N$ is assumed to be 8000 , and the capacity $s$ of the bottleneck is 4000 vehicles per hour.

Fig. 6a shows the departure rate of commuters during the morning peak period. It can be seen that the departure rate over time comprises a continuous and monotonically decreasing curve, which is different from the departure rate curve of the traditional Vickrey's bottleneck model, which is piecewise constant (see Fig. 1a). Fig. 6b displays the results of the cumulative departures from home and the cumulative arrivals at the workplace during the morning commute. It shows that the cumulative departure curve is nonlinear, continuous and differentiable, unlike the Vickrey's bottleneck model, in which the cumulative departure curve is piecewise linear and non-differentiable (see Fig. 1b).

Fig. 6c shows that the queuing delay time at the bottleneck during the morning commute first increases and then decreases, and the maximum queuing delay of 0.319 hour occurs at $\hat{t}=0.26$, in terms of Eq. (33). This is because when $t<\hat{t}=0.26$, the departure rate $r(t)$ is greater than the bottleneck capacity of $s=4000$ vehicles per hour. When $t>\hat{t}=0.26$, the departure rate is less than the bottleneck capacity s, as shown in Fig. 6a. Fig. 6c also shows that the queuing delay curve in the activity-based bottleneck model with the linear marginal activity utilities is also nonlinear, continuous and differentiable. However, it is piecewise linear and non-differentiable for the traditional Vickrey's bottleneck model (see Fig. 1c). This further illustrates the results in Proposition 3 and Remark 3.

\section{Step tolling with homogeneous preferences}

In this section, we investigate the step tolling problem in the activity-based bottleneck model with linear marginal activity utilities under homogeneous preferences. To examine the efficiency of a step toll scheme in removing the queue at the bottleneck, the social optimum (i.e., first-best) tolling scheme is used as the benchmark. 


\subsection{Social optimum tolling scheme}

The social optimum refers to a situation in which the total scheduling cost of commuters is minimized. The following proposition reveals the relationship between the social optimum and user equilibrium.

Proposition 4. For the activity-based bottleneck model with linear marginal activity utilities, when the queuing delay at the bottleneck is just replaced by a time-varying toll, the no-toll user equilibrium is automatically driven to the social optimum.

Proof. We need to prove that there is a time-varying toll such that marginal social cost equals marginal private cost. Suppose that a time-varying toll $\tau(t)$ is imposed and the resultant queuing delay time at the bottleneck at any time $t$ is zero, i.e., $T(t)=0$. From Eq. (24), when $T(t)=0$, the travel cost function $C(t)$ with an imposed toll $\tau(t)$ becomes

$$
\begin{aligned}
C(t) & =\int_{t}^{t^{* *}} h(t) d t+\int_{t^{* *}}^{t} w(t) d t+\tau(t) \\
& =\frac{1}{2}(\lambda-\delta) t^{2}-(\xi-\mu) t+\frac{(\xi-\mu)^{2}}{2(\lambda-\delta)}+\tau(t), \quad t \in\left[t_{q}, t_{q^{\prime}}\right],
\end{aligned}
$$

where $\tau(t)$ denotes the congestion toll at the bottleneck at time $t$ during the morning peak.

Note that the first (or last) commuter who departs from home faces no queue, and thus, the associated congestion toll for the first (or last) commuter is zero, i.e., $\tau\left(t_{q}\right)=\tau\left(t_{q^{\prime}}\right)=0$. The resultant travel cost can, therefore, be given by $C\left(t_{q}\right)=C\left(t_{q^{\prime}}\right)=\frac{N^{2}}{8 s^{2}}(\lambda-\delta)$ according to Eq. $(30)$.

At the equilibrium, $C\left(t_{q}\right)=C\left(t_{q^{\prime}}\right)=C(t)$. From Eq. (35), the time-varying toll $\tau(t)$ can be given by

$$
\tau(t)=-\frac{1}{2}(\lambda-\delta) t^{2}+(\xi-\mu) t-\frac{(\xi-\mu)^{2}}{2(\lambda-\delta)}+\frac{N^{2}}{8 s^{2}}(\lambda-\delta), \quad t \in\left[t_{q}, t_{q^{\prime}}\right] .
$$

Eq. (36) implies that there is a time-varying congestion toll $\tau(t)$, which ensures that the 
travel costs of all commuters are equal to $C\left(t_{q}\right)$ or $C\left(t_{q^{\prime}}\right)$. As a result, the total social cost (SC) for all commuters is

$$
\begin{aligned}
\mathrm{SC} & =\int_{t_{q}}^{t_{t^{q^{\prime}}}}(C(t)-\tau(t)) s d t=\int_{t_{q}}^{t_{q^{\prime}}}\left(\frac{1}{2}(\lambda-\delta) t^{2}-(\xi-\mu) t+\frac{(\xi-\mu)^{2}}{2(\lambda-\delta)}\right) s d t \\
& =\frac{N^{3}}{24 s^{2}}(\lambda-\delta) .
\end{aligned}
$$

It should be pointed out that the congestion toll is not included in the total social cost. This is because the payment of toll implies only a transfer of money from road users to the authority within the system and not a deadweight loss.

Consequently, the marginal social cost $d \mathrm{SC} / d N$ can be calculated as

$$
\frac{d S C}{d N}=\frac{N^{2}}{8 s^{2}}(\lambda-\delta)=C\left(t_{q}\right)=C\left(t_{q^{\prime}}\right) .
$$

Eq. (38) shows that the marginal social cost exactly equals the marginal private cost $C\left(t_{q}\right)$ or $C\left(t_{q^{\prime}}\right)$, implying that the system optimum state is reached. This completes the proof of this proposition.

From Proposition 4, the congestion toll under the social optimum tolling scheme directly substitutes for the queuing delay at the bottleneck, and thus the queuing delay is zero. Apparently, in both the social optimum and the no-toll equilibrium, the capacity of the bottleneck is fully utilized during the morning peak, i.e., the departure rate equals the capacity of the bottleneck. Moreover, the travel costs are the same for the first and last commuters for both the social optimum and the no-toll equilibrium. Thus, for a given number of commuters, the start and end times of the morning peak period and the cumulative arrivals under the social optimum are the same as those under the no-toll equilibrium.

Remark 4. Since the time-varying toll $\tau(t)$ in Eq. (36) directly replaces the queuing delay at the bottleneck, $\tau(t)$ also represents the queuing delay cost at the bottleneck at time $t . T(t)$ in Eq. (32) represents the queuing delay time at the bottleneck at time $t$. Thereby, $\tau(t)$ is measured in monetary unit, whereas $T(t)$ is measured in time unit. From Eqs. (32) and (36), one can easily derive the following relationship: 


$$
\frac{\tau(t)}{T(t)}=w(t)+\frac{\lambda T(t)}{2}, t \in\left[t_{q}, t_{q^{\prime}}\right]
$$

Eq. (39) shows that a marginal decrease in the queuing delay time requires an increase in the toll by $w(t)+\frac{\lambda T(t)}{2}$. The value of the queuing delay time is thus larger than the marginal work utility $w(t)$.

For the bottleneck model with linear marginal activity utilities, the social optimum time-varying congestion toll $\tau(t)$ in Eq. (36) represents a parabolic curve with symmetric axis $t^{* *}$ and maximum toll $\tau_{\max }$, as follows.

$$
\left\{\begin{array}{l}
t^{* *}=\frac{\xi-\mu}{\lambda-\delta} \\
\tau_{\max }=\frac{N^{2}}{8 s^{2}}(\lambda-\delta) .
\end{array}\right.
$$

Note that the area of the diagram below the time-varying congestion toll curve $\tau(t)$ equals the bottleneck queuing delay cost under the no-toll equilibrium, represented as $\Phi^{\mathrm{NT}}$. Thereby, the total queuing delay cost $\Phi^{\mathrm{NT}}$, which equals the total toll revenue, can be given by

$$
\begin{aligned}
\Phi^{\mathrm{NT}} & =\int_{t_{q}}^{t_{q^{\prime}}} \tau(t) s d t=\int_{\frac{\xi-\mu}{\lambda-\delta}-\frac{N}{2 s}}^{\frac{\xi-\mu}{\lambda-\delta}+\frac{N}{2 s}}\left(-\frac{1}{2}(\lambda-\delta) t^{2}+(\xi-\mu) t-\frac{(\xi-\mu)^{2}}{2(\lambda-\delta)}+\frac{N^{2}}{8 s^{2}}(\lambda-\delta)\right) s d t \\
& =\frac{N^{3}}{12 s^{2}}(\lambda-\delta),
\end{aligned}
$$

where the superscript "NT" represents the no-toll case.

The social-optimum congestion toll $\tau(t)$ changes continuously over time, and its implementation thus becomes prohibitive due to drivers' cognitive barrier. Therefore, a step toll scheme has been suggested as a substitute or approximation to the social-optimum time-varying toll scheme. In the step toll scheme, the study period is discretized into small intervals, and the toll takes different values over these discrete time intervals but remains constant within each interval. Although the step tolling system cannot remove queues completely, it can easily be implemented in practice, such as the London's single-step tolling system. It may, thus, be more acceptable than the social-optimum time-varying toll scheme. 


\subsection{Step tolling schemes}

The optimal step toll scheme aims to determine the optimal tolling time points for each step and the corresponding toll level. In this subsection, we focus on the optimal step toll scheme with linear marginal activity utilities. A step toll structure is called an optimal scheme if and only if it removes the largest proportion of the total bottleneck queuing delay. Note that maximizing queuing removal under the toll scheme is equivalent to maximizing the toll revenue, which requires that the optimal step tolls are inscribed in the concave social optimum time-varying toll curve (see Eq. (36)). If the step toll is beyond the social optimum time-varying toll during a time period, then it will impose a higher commuting cost to those who depart during that time period than the no-toll equilibrium cost due to an excessively high toll. As a result, the equilibrium will be inefficient. Consequently, the maximum toll revenue can be achieved if and only if the optimal step tolls are inscribed in the social optimum time-varying toll curve. In the following, single-step and multistep toll schemes are in turn presented.

\subsubsection{Single-step toll}

The single-step toll scheme is defined by its toll level $\rho_{1}$ and the times at which it is turned on $\left(t_{1}^{+}\right)$and off $\left(t_{1}^{-}\right)$, where the subscript " 1 " represents the single-step toll scheme. Obviously, $\rho_{1}=\tau\left(t_{1}^{+}\right)=\tau\left(t_{1}^{-}\right)$holds for the single-step toll scheme. Because the social optimum time-varying congestion toll curve $\tau(t)$ is symmetric with regard to $t=t^{* *}$ (see Eq. (40) and Fig. 7), the tolling start time $t_{1}^{+}$and end time $t_{1}^{-}$are also symmetric with regard to $t=t^{* *}$, implying $t^{* *}-t_{1}^{-}=t_{1}^{+}-t^{* *}$ or $t_{1}^{+}=2 t^{* *}-t_{1}^{-}$. The goal of the single-step toll scheme is to maximize queuing removal by designing the combination of $t_{1}^{+}$and $t_{1}^{-}$, subject to $\rho_{1}=\tau\left(t_{1}^{+}\right)=\tau\left(t_{1}^{-}\right)$and $t_{1}^{+}=2 t^{* *}-t_{1}^{-}$, as illustrated in Fig. 7. Note that the area of the diagram below the time-varying congestion toll curve $\tau(t)$ equals the queuing delay cost at the bottleneck. Accordingly, maximizing queuing removal under the toll scheme is equivalent to maximizing the toll revenue, which is the area of the rectangle inscribed in the optimal time-varying toll curve, expressed as

$$
\max \Lambda_{1}\left(\rho_{1}, t_{1}^{-}, t_{1}^{+}\right)=\left(t_{1}^{-}-t_{1}^{+}\right) \rho_{1} s,
$$




$$
\text { s.t. }\left\{\begin{array}{l}
\rho_{1}=\tau\left(t_{1}^{+}\right)=\tau\left(t_{1}^{-}\right) \\
t_{1}^{+}=2 t^{* *}-t_{1}^{-}
\end{array}\right.
$$

Substituting Eqs. (36) and (43) into Eq. (42) yields

$$
\max \Lambda_{1}\left(t_{1}^{-}\right)=2 s\left(t_{1}^{-}-\frac{\xi-\mu}{\lambda-\delta}\right)\left(-\frac{1}{2}(\lambda-\delta)\left(t_{1}^{-}\right)^{2}+(\xi-\mu) t_{1}^{-}-\frac{(\xi-\mu)^{2}}{2(\lambda-\delta)}+\frac{N^{2}}{8 s^{2}}(\lambda-\delta)\right) .
$$

From the first-order optimality condition of maximization problem (44), one obtains

$$
t_{1}^{-}=\frac{\xi-\mu}{\lambda-\delta}+\frac{\sqrt{3}}{6} \frac{N}{s}, \text { and } t_{1}^{+}=\frac{\xi-\mu}{\lambda-\delta}-\frac{\sqrt{3}}{6} \frac{N}{s} .
$$

Substituting Eq. (45) into $\rho_{1}=\tau\left(t_{1}^{+}\right)=\tau\left(t_{1}^{-}\right)$, we have

$$
\rho_{1}=\frac{N^{2}}{12 s^{2}}(\lambda-\delta)=\frac{2}{3} \tau_{\max }
$$

where $\tau_{\max }=\frac{N^{2}}{8 s^{2}}(\lambda-\delta)$, as given in Eq. (40).

The resultant maximum queuing removal is

$$
\Lambda_{1, \max }=\frac{\sqrt{3}}{36} \frac{N^{3}}{s^{2}}(\lambda-\delta)=\frac{\sqrt{3}}{3} \Phi^{\mathrm{NT}},
$$

where the queuing delay cost $\Phi^{\mathrm{NT}}$ under the no-toll equilibrium is given by Eq. (41).

The following proposition summarizes the results under the single-step toll scheme.

Proposition 5. For the activity-based bottleneck model with linear marginal activity utilities, the optimal single-step toll is $\frac{2}{3}(\approx 0.667)$ of the maximum optimal time-varying toll and can eliminate $\frac{\sqrt{3}}{3}(\approx 0.577)$ of the total queuing delay that exists under the no-toll equilibrium.

Remark 5. According to Lemma 1 and Proposition 5, the optimal single-step tolls $\rho_{1}$ are, respectively, $\frac{1}{2}$ and $\frac{2}{3}$ of the corresponding maximum optimal time-varying toll $\tau_{\max }$ for the activity-based bottleneck models with constant marginal utilities (i.e., Laih model) and 
with linear marginal utilities (proposed in this paper). They can eliminate $\frac{1}{2}$ and $\frac{\sqrt{3}}{3}$ of the associated total queuing delay that exists under the no-toll equilibrium. This means that compared to the activity-based bottleneck model with linear marginal activity utilities, the activity-based bottleneck model with constant marginal activity utilities (i.e., Laih model) underestimates the optimal single-step toll solution in terms of ratio $\frac{\rho_{1}}{\tau_{\max }}$ and the role of the single-step toll in removing the bottleneck queue in terms of the queuing removal rate $Q R R$, with $Q R R=\frac{\Lambda_{1, \max }}{\Phi^{\mathrm{NT}}}$, i.e., the proportion of the removed bottleneck queuing delay to the total queuing delay under the no-toll equilibrium.

\subsubsection{Multistep toll}

The goal of the multistep (e.g., $m$-step) toll scheme is to determine the optimal tolling time points $t_{i}^{+}$and $t_{i}^{-} \quad(i=1,2, \ldots, m)$ and the corresponding toll levels $\rho_{i}$ and $\rho_{i}^{\prime}$, where $\rho_{i}=\tau\left(t_{i}^{+}\right)$and $\rho_{i}^{\prime}=\tau\left(t_{i}^{-}\right)$for any $i=1,2, \ldots, m$, as shown in Fig. 8. In the following, we first present an important finding, i.e., the property of the optimal multistep toll scheme.

Proposition 6. The optimal multistep toll scheme with linear marginal activity utilities must follow a symmetric (or shoulder) toll structure with $t=t^{* *}$ as the symmetric axis, i.e., $t^{* *}-t_{i}^{-}=t_{i}^{+}-t^{* *}$ and $\rho_{i}=\rho_{i}^{\prime}, \quad i=1,2, \ldots, m$.

The proof of Proposition 6 is provided in Appendix B. This proposition shows that the optimal tolling time points $t_{i}^{+}$and $t_{i}^{-}$of the ith step $(i=1,2, \ldots, m)$ are symmetric with regard to $t=t^{* *}$ and that the associated toll levels $\rho_{i}$ and $\rho_{i}^{\prime}$ at $t_{i}^{+}$and $t_{i}^{-}$are equal. In what follows, we denote the $i$ th step toll level as $\rho_{i}$, where $\rho_{i}=\rho_{i}^{\prime}=\tau\left(t_{i}^{+}\right)=\tau\left(t_{i}^{-}\right)$for any step $i=1,2, \ldots, m$.

We now look at the multistep toll problem, beginning with the two-step toll, as shown in Fig. 9. Similar to the single-step toll scheme, the two-step toll scheme aims to determine the toll levels $\rho_{1}$ and $\rho_{2}$ and the charging time points $t_{1}^{+}, t_{1}^{-}, t_{2}^{+}$, and $t_{2}^{-}$to maximize the 
queuing removal (or the total toll revenue), which is the sum of the areas of the rectangles inscribed in the optimal time-varying toll curve, i.e.,

$$
\begin{aligned}
& \max \Lambda_{2}\left(\rho_{1}, \rho_{2}, t_{1}^{-}, t_{1}^{+}, t_{2}^{-}, t_{2}^{+}\right)=\rho_{1} s\left(t_{1}^{-}-t_{1}^{+}\right)+\rho_{2} s\left(t_{1}^{+}-t_{2}^{+}\right)+\rho_{2} s\left(t_{2}^{-}-t_{1}^{-}\right), \\
& \text {s.t. }\left\{\begin{array}{l}
\rho_{1}=\tau\left(t_{1}^{+}\right)=\tau\left(t_{1}^{-}\right), \\
\rho_{2}=\tau\left(t_{2}^{+}\right)=\tau\left(t_{2}^{-}\right), \\
t_{1}^{+}=2 t^{* *}-t_{1}^{-}, \\
t_{2}^{+}=2 t^{* *}-t_{2}^{-} .
\end{array}\right.
\end{aligned}
$$

Substituting Eqs. (36) and (49) into Eq. (48) yields the following unconstrained optimization problem.

$$
\begin{aligned}
& \max \Lambda_{2}\left(t_{1}^{-}, t_{2}^{-}\right)=2 \rho_{1} s\left(t_{1}^{-}-t^{* *}\right)+2 \rho_{2} s\left(t_{2}^{-}-t_{1}^{-}\right) \\
& =2 s\left(t_{1}^{-}-\frac{\xi-\mu}{\lambda-\delta}\right)\left(-\frac{1}{2}(\lambda-\delta)\left(t_{1}^{-}\right)^{2}+(\xi-\mu) t_{1}^{-}-\frac{(\xi-\mu)^{2}}{2(\lambda-\delta)}+\frac{N^{2}}{8 s^{2}}(\lambda-\delta)\right) \\
& +2 s\left(t_{2}^{-}-t_{1}^{-}\right)\left(-\frac{1}{2}(\lambda-\delta)\left(t_{2}^{-}\right)^{2}+(\xi-\mu) t_{2}^{-}-\frac{(\xi-\mu)^{2}}{2(\lambda-\delta)}+\frac{N^{2}}{8 s^{2}}(\lambda-\delta)\right) .
\end{aligned}
$$

From the first-order optimality conditions of the maximization problem (50), we obtain

$$
\left\{\begin{array} { l } 
{ t _ { 1 } ^ { - } = \frac { \xi - \mu } { \lambda - \delta } + \frac { 1 } { 2 \sqrt { 9 - 2 \sqrt { 3 } } } \frac { N } { s } , } \\
{ t _ { 2 } ^ { - } = \frac { \xi - \mu } { \lambda - \delta } + \frac { \sqrt { 3 } } { 2 \sqrt { 9 - 2 \sqrt { 3 } } } \frac { N } { s } , }
\end{array} \quad \left\{\begin{array} { l } 
{ t _ { 1 } ^ { + } = \frac { \xi - \mu } { \lambda - \delta } - \frac { 1 } { 2 \sqrt { 9 - 2 \sqrt { 3 } } } \frac { N } { \mathrm { s } } , } \\
{ t _ { 2 } ^ { + } = \frac { \xi - \mu } { \lambda - \delta } - \frac { \sqrt { 3 } } { 2 \sqrt { 9 - 2 \sqrt { 3 } } } \frac { N } { \mathrm { s } } , }
\end{array} \text { and } \left\{\begin{array}{l}
\rho_{1}=\frac{8-2 \sqrt{3}}{9-2 \sqrt{3}} \tau_{\text {max }}, \\
\rho_{2}=\frac{6-2 \sqrt{3}}{9-2 \sqrt{3}} \tau_{\max } .
\end{array}\right.\right.\right.
$$

The resultant maximum queuing removal is

$$
\Lambda_{2, \max }=\frac{(3 \sqrt{3}-2) \sqrt{9-2 \sqrt{3}}}{31-12 \sqrt{3}} \frac{N^{3}}{12 s^{2}}(\lambda-\delta)=\frac{(3 \sqrt{3}-2) \sqrt{9-2 \sqrt{3}}}{31-12 \sqrt{3}} \Phi^{\mathrm{NT}} .
$$

Proposition 7. For the activity-based bottleneck model with linear marginal activity utilities, the optimal two-step tolls $\rho_{1}$ and $\rho_{2}$, respectively, are $\frac{8-2 \sqrt{3}}{9-2 \sqrt{3}}(\approx 0.819)$ and $\frac{6-2 \sqrt{3}}{9-2 \sqrt{3}}(\approx 0.458)$ of the maximum optimal time-varying toll and can eliminate $\frac{(3 \sqrt{3}-2) \sqrt{9-2 \sqrt{3}}}{31-12 \sqrt{3}}(\approx 0.736)$ of the total queuing delay that exists under the no-toll 
equilibrium.

Remark 6. For the convenience of the readers, we have summarized in Table 1 the optimal solutions for the single-step and two-step toll schemes for the activity-based bottleneck model with linear marginal activity utilities. It shows that for a given number of steps, the activity-based bottleneck model with constant marginal utilities (i.e., traditional bottleneck model) underestimates the optimal step toll solution in terms of the proportion of the optimal step toll solution to the maximum optimal time-varying toll and the role of the step toll scheme in terms of the queuing removal rate, compared to the activity-based bottleneck model with linear marginal utilities. This means that for a given number of steps, the efficacy of a step toll is higher in the activity-based bottleneck model with linear marginal utilities than in that with constant marginal utilities in terms of the queuing removal rate.

Similar to the two-step tolling problem, one can design an $m$-step toll scheme (see Fig. 8), in which the toll levels $\rho_{i} \quad(i=1,2, \ldots, m)$ and the charging time points $t_{i}^{+}$and $t_{i}^{-}$ $(i=1,2, \ldots, m)$ are determined to maximize the queuing removal subject to $\rho_{i}=\tau\left(t_{i}^{+}\right)=\tau\left(t_{i}^{-}\right)$ and $t_{i}^{+}=2 t^{* *}-t_{i}^{-}, i=1,2, \ldots, m$. The $m$-step tolling model can be formulated as the following maximization problem.

$$
\begin{aligned}
& \max \Lambda_{m}\left(\rho_{1}, \rho_{2}, \ldots, \rho_{m}, t_{1}^{-}, t_{2}^{-}, \ldots, t_{m}^{-}, t_{1}^{+}, t_{2}^{+}, \ldots, t_{m}^{+}\right)=\rho_{1} s\left(t_{1}^{-}-t_{1}^{+}\right)+\sum_{i=2}^{m} \rho_{i} s\left(t_{i}^{-}-t_{i-1}^{-}\right)+\sum_{i=2}^{m} \rho_{i} s\left(t_{i-1}^{+}-t_{i}^{+}\right), \\
& \text {s.t. } \quad\left\{\begin{array}{l}
\rho_{i}=\tau\left(t_{i}^{+}\right)=\tau\left(t_{i}^{-}\right), i=1,2, \ldots, m, \\
t_{i}^{+}=2 t^{* *}-t_{i}^{-}, i=1,2, \ldots, m .
\end{array}\right.
\end{aligned}
$$

Substituting Eqs. (36) and (54) into (53) yields the following unconstrained maximization problem.

$$
\max \Lambda_{m}\left(t_{1}^{-}, t_{2}^{-}, \ldots, t_{m}^{-}\right)=2 \rho_{1} s\left(t_{1}^{-}-t^{* *}\right)+\sum_{i=2}^{m} 2 \rho_{i} s\left(t_{i}^{-}-t_{i-1}^{-}\right) .
$$

The first-order optimality condition of the maximization problem (55) is 


$$
\left\{\begin{array}{l}
3\left(t_{1}^{-}\right)^{2}-\left(t_{2}^{-}\right)^{2}-\frac{2(\xi-\mu)}{\lambda-\delta}\left(3 t_{1}^{-}-t_{2}^{-}-\frac{\xi-\mu}{\lambda-\delta}\right)=0 \\
3\left(t_{i}^{-}\right)^{2}-2 t_{i-1}^{-} t_{i}^{-}-\left(t_{i+1}^{-}\right)^{2}-\frac{2(\xi-\mu)}{\lambda-\delta}\left(2 t_{i}^{-}-t_{i-1}^{-}-t_{i+1}^{-}\right)=0, i=2,3, \ldots, m-1 \\
3\left(t_{m}^{-}\right)^{2}-2 t_{m-1}^{-} t_{m}^{-}-\frac{N^{2}}{4 s^{2}}+\left(\frac{\xi-\mu}{\lambda-\delta}\right)^{2}-\frac{2(\xi-\mu)}{\lambda-\delta}\left(2 t_{m}^{-}-t_{m-1}^{-}\right)=0
\end{array}\right.
$$

Solving the system of equations (56), one can obtain the optimal solutions of the decision variables $\rho_{i}, t_{i}^{+}$, and $t_{i}^{-}(i=1,2, \ldots, m)$ and thus the corresponding maximum queuing removal $\Lambda_{m, \max }$ and the queuing removal rate $\frac{\Lambda_{m, \max }}{\Phi^{\mathrm{NT}}}$.

\subsection{An example of three-step tolls}

Suppose that a three-step toll scheme is applied to the bottleneck system, and the marginal utility functions for home and work activities are linear, as defined in Eqs. (22) and (23). The input data for all model parameters are the same as the example in Section 3.4. Substituting the parameter values into Eq. (56), we obtain the solutions of the three-step toll scheme as follows: $\rho_{3}=\$ 5.91, \quad \rho_{2}=\$ 11.06, \quad \rho_{1}=\$ 15.12, \quad t_{3}^{+}=-0.31, \quad t_{2}^{+}=-0.10, \quad t_{1}^{+}=0.15$, $t_{1}^{-}=0.85, t_{2}^{-}=1.10$, and $t_{3}^{-}=1.31$, as shown in Fig. 10. The total queuing delay cost $\Phi^{\mathrm{NT}}$ that exists under the no-toll equilibrium is $\$ 91413$. The maximum queuing delay cost removal $\Lambda_{3, \max }$ is $\$ 73953$. This means that the queuing removal rate $\frac{\Lambda_{3, \max }}{\Phi^{\mathrm{NT}}}$ equals 0.809 . Compared to the queuing removal rates of the single-step and two-step toll schemes (i.e., 0.577 and 0.736), the queuing removal rate of the three-step toll scheme further increases. This is consistent with our intuition, namely as the number of steps increases, the discretized step tolling schemes are gradually close to the continuous time-varying tolling scheme. However, it should be mentioned that too many steps may add toll collection cost and confuse travelers due to their bounded rationality such that they may not be able to respond effectively to the price signals. Therefore, there exists a trade-off between the incremental benefits of reducing travel costs by adding toll steps and the incremental toll collection and driver compliance costs that would be incurred. The optimal number of steps may thus exist. The optimization problem of the number of steps makes an interesting and important research topic, but is outside the scope of this paper and thus left for future study. 


\section{Incorporating preference heterogeneity}

In the previous sections, we have addressed the step tolling problem in the bottleneck model with homogeneous preferences. In this section, we extend it to the case of heterogeneous preferences. Commuters are grouped according to their heterogeneous preferences, i.e., the marginal utility functions for home/work activities. For simplification purpose, in the paper we focus on two groups, 1 and 2. Their linear marginal utility functions for home and work activities are, respectively, specified as

$$
\left\{\begin{array}{l}
h_{g}(t)=\xi_{g}+\delta_{g} t \\
w_{g}(t)=\mu_{g}+\lambda_{g} t
\end{array}, g=1,2\right.
$$

where $g$ denotes a group of commuters. For any group $g$, the parameters $\xi_{g}, \mu_{g}$, and $\lambda_{g}$ are positive, and the parameter $\delta_{g}$ is negative.

Let $N_{g}$ be the number of commuters in group $g$ and $N$ be the total number of commuters in the system, with

$$
N_{1}+N_{2}=N \text {. }
$$

\subsection{No-toll equilibrium}

We first analyze the no-toll equilibrium with heterogeneous commuters. By the equilibrium's definition, at the equilibrium, no commuter can reduce his/her trip cost by changing choice of departure time. This means that all members in a group incur the same trip cost for their chosen departure times, and equal or higher costs at any other times.

According to the isocost queuing delay curve under homogeneous preferences (see Fig. 6c), one can depict the isocost queuing delay curves with considering commuters' heterogeneous preferences. For illustration purpose, Fig. 11 displays the case of two groups, in which group 2 has a higher marginal work utility than group 1 (i.e., $w_{1} \leq w_{2}$ ), but both groups have the same marginal home activity utility. Thereby, the isocost queuing delay curve for group 2 is on the left of that for group 1 in terms of the axis $t_{g}^{* *}=\frac{\xi_{g}-\mu_{g}}{\lambda_{g}-\delta_{g}}$ (cf. Eq. (40)). It can be seen that the isocost queuing delay curves for the two groups may not intersect (Fig. 11a) or 
intersect (Fig. 11b). Apparently, the case without overlap can be seen as a simple combination of the (separate) isocost curves of different groups. Thus, all properties under the homogeneous case, as presented in previous sections, still hold. In the following, we consider only the case that the isocost queuing delay curves for different groups intersect.

Note that at any given time, all commuters face the same queue. This implies that the isocost queuing delay curve with heterogeneous groups is the upper envelope of all groups' isocost queuing delay curves, as shown in bold in Fig. 11b. Otherwise, any commuter in other group could reduce its travel cost by changing its departure time. The equilibrium frontier means that each departure slot is allocated to that group which is willing to pay the most for it, in terms of queuing delay. Note that as the marginal work utility (i.e., $\mu_{g}$ or $\lambda_{g}$ ) of a specific group increases, the axis $t_{g}^{* *}=\frac{\xi_{g}-\mu_{g}}{\lambda_{g}-\delta_{g}}$ of that group moves towards the left, and thus the isocost queuing delay curve for that group also moves towards the left. Similarly, given the marginal work utility (i.e., $\mu_{g}$ and $\lambda_{g}$ ), one can easily find that as the marginal home activity utility (i.e., $\xi_{g}$ or $\delta_{g}$ ) of a specific group increases, the isocost queuing delay curve for that group moves towards the right. We thus have the following property for the departure time choice equilibrium of heterogeneous groups.

Proposition 8. At the equilibrium, groups sequentially depart in order of decreasing marginal work activity utility or increasing marginal home activity utility.

Proposition 8 implies that if group 2 has a higher marginal work (home) activity utility than group 1, then group 2 (group 1) is the first to depart, and then group 1 (group 2). There exists a boundary time between group 1's and group 2's departures, i.e. the intersection between the isocost queuing delay curves of the two groups, which is represented as $t_{12}$. In Fig. 11, group 2 departs at interval $\left[t_{q}, t_{12}\right]$, while group 1 departs at interval $\left[t_{12}, t_{q^{\prime}}\right]$, that is,

$$
\left\{\begin{array}{l}
t_{12}-t_{q}=N_{2} / s \\
t_{q^{\prime}}-t_{12}=N_{1} / s
\end{array}\right.
$$

At the equilibrium, the commuters from an identical group should have the same travel cost regardless of their departure times. We thus have 
$C_{1}\left(t_{12}\right)=C_{1}\left(t_{q^{\prime}}\right)$, where $\left\{\begin{array}{l}C_{1}\left(t_{12}\right)=-\frac{\delta_{1}}{2} t_{12}^{2}-\left(\xi_{1}-\mu_{1}\right) t_{12}+\mu_{1} T\left(t_{12}\right)+\frac{\lambda_{1}}{2}\left(t_{12}+T\left(t_{12}\right)\right)^{2}+\frac{\left(\xi_{1}-\mu_{1}\right)^{2}}{2\left(\lambda_{1}-\delta_{1}\right)}, \\ C_{1}\left(t_{q^{\prime}}\right)=\frac{1}{2}\left(\lambda_{1}-\delta_{1}\right) t_{q^{\prime}}^{2}-\left(\xi_{1}-\mu_{1}\right) t_{q^{\prime}}+\frac{\left(\xi_{1}-\mu_{1}\right)^{2}}{2\left(\lambda_{1}-\delta_{1}\right)} .\end{array}\right.$
$C_{2}\left(t_{12}\right)=C_{2}\left(t_{q}\right)$, where $\left\{\begin{array}{l}C_{2}\left(t_{12}\right)=-\frac{\delta_{2}}{2} t_{12}^{2}-\left(\xi_{2}-\mu_{2}\right) t_{12}+\mu_{2} T\left(t_{12}\right)+\frac{\lambda_{2}}{2}\left(t_{12}+T\left(t_{12}\right)\right)^{2}+\frac{\left(\xi_{2}-\mu_{2}\right)^{2}}{2\left(\lambda_{2}-\delta_{2}\right)}, \\ C_{2}\left(t_{q}\right)=\frac{1}{2}\left(\lambda_{2}-\delta_{2}\right) t_{q}^{2}-\left(\xi_{2}-\mu_{2}\right) t_{q}+\frac{\left(\xi_{2}-\mu_{2}\right)^{2}}{2\left(\lambda_{2}-\delta_{2}\right)} .\end{array}\right.$

It should be mentioned that the expressions for $C_{1}\left(t_{12}\right), C_{2}\left(t_{12}\right), C_{1}\left(t_{q^{\prime}}\right)$, and $C_{2}\left(t_{q}\right)$ in Eqs. (60) and (61) are directly from Eqs. (24)-(26). Solving the system of Eqs. (59)-(61), one can obtain the no-toll equilibrium solutions of $t_{q}, t_{q^{\prime}}, t_{12}$, and $T\left(t_{12}\right)$.

\subsection{Step tolling with heterogeneous groups}

In this section, we examine the effects of the preference heterogeneity of commuters on the step tolling. Without loss of generality, in what follows we consider the heterogeneity in the marginal work utility only. That is, the parameters $\mu_{g}$ and $\lambda_{g}$ in Eq. (57) are different across commuter groups, but the parameters $\xi_{g}$ and $\delta_{g}$ are the same for different groups. Hence, the subscripts for $\xi$ and $\delta$ can be removed, i.e., $\xi_{1}=\xi_{2}=\xi$ and $\delta_{1}=\delta_{2}=\delta$. For the heterogeneity in the marginal home activity utility, one can easily derive its results in the same way. In order to save space, they are not shown here. In the following, we first present the social optimum toll and then determine the optimal step toll for the heterogeneity in the marginal work utility $w$. To do so, it is assumed that group 2 has a higher marginal work utility than group 1 (i.e., $w_{1} \leq w_{2}$ ).

\subsubsection{Social optimum tolling scheme}

Similar to the homogeneous case, the social optimum with heterogeneous groups involves no queuing because the queuing delay at the bottleneck is directly displaced by the toll. Under the assumption of $w_{1} \leq w_{2}$, group 2 departs earlier than group 1 in terms of Proposition 8 . Specifically, group 2 departs at $\left[t_{q}, t_{12}\right]$, and group 1 departs at $\left[t_{12}, t_{q^{\prime}}\right]$, as shown in Fig. $11 \mathrm{~b}$. 
The equilibrium with time-varying toll requires that the travel cost of all commuters in group 1 equals the last commuter's travel cost, i.e.,

$$
\begin{aligned}
C_{1}\left(t_{q^{\prime}}\right) & =\int_{t}^{t_{1}^{* *}} h_{1}(t) d t+\int_{t_{1}^{* *}}^{t} w_{1}(t) d t+\tau_{1}(t) \\
& =\frac{1}{2}\left(\lambda_{1}-\delta\right) t^{2}-\left(\xi-\mu_{1}\right) t+\frac{\left(\xi-\mu_{1}\right)^{2}}{2\left(\lambda_{1}-\delta\right)}+\tau_{1}(t), \quad t \in\left[t_{12}, t_{q^{\prime}}\right],
\end{aligned}
$$

where $\tau_{1}(t)$ is the congestion toll at the bottleneck at time $t \in\left[t_{12}, t_{q^{\prime}}\right]$.

Substituting $C_{1}\left(t_{q^{\prime}}\right)$ in Eq. (60) into Eq. (62) yields the optimal isocost time-varying toll for group 1 between $t_{12}$ and $t_{q^{\prime}}$ as follows.

$$
\tau_{1}(t)=\frac{1}{2}\left(\lambda_{1}-\delta\right)\left(t_{q^{\prime}}^{2}-t^{2}\right)-\left(\xi-\mu_{1}\right)\left(t_{q^{\prime}}-t\right), \quad t \in\left[t_{12}, t_{q^{\prime}}\right]
$$

Similarly, for group 2, the travel cost of all commuters equals the first commuter's travel cost, i.e.,

$$
\begin{aligned}
C_{2}\left(t_{q}\right) & =\int_{t}^{t_{2}^{* *}} h_{2}(t) d t+\int_{t_{2}^{* *}}^{t} w_{2}(t) d t+\tau_{2}(t) \\
& =\frac{1}{2}\left(\lambda_{2}-\delta\right) t^{2}-\left(\xi-\mu_{2}\right) t+\frac{\left(\xi-\mu_{2}\right)^{2}}{2\left(\lambda_{2}-\delta\right)}+\tau_{2}(t), \quad t \in\left[t_{q}, t_{12}\right]
\end{aligned}
$$

where $\tau_{2}(t)$ is the congestion toll at the bottleneck at time $t \in\left[t_{q}, t_{12}\right]$.

Substituting $C_{2}\left(t_{q}\right)$ in Eq. (61) into Eq. (64), one obtains the optimal isocost time-varying toll for group 2 between $t_{q}$ and $t_{12}$ as below.

$$
\tau_{2}(t)=\frac{1}{2}\left(\lambda_{2}-\delta\right)\left(t_{q}^{2}-t^{2}\right)-\left(\xi-\mu_{2}\right)\left(t_{q}-t\right), \quad t \in\left[t_{q}, t_{12}\right]
$$

The social optimum time-varying toll curves for groups 1 and 2, which are defined in Eqs. (63) and (65), are depicted in bold in Fig. 12. It shows that the social optimum with heterogeneous groups can be achieved with a piecewise quadratic time-varying tolling scheme that exactly replaces the bottleneck queuing delay costs.

\subsubsection{Step tolling scheme}


The continuously changing charges, as expressed in Eqs. (63) and (65), are difficult to be implemented in real-world applications. Thus, a second-best step tolling scheme is usually adopted in practice, which is presented as follows. For ease of presentation, again we consider two groups of commuters, with $w_{1} \leq w_{2}$. In order to derive a closed-form solution, we limit our attentions to a single-step tolling scheme for each group in this paper. The single-step toll structure to be determined for a two-group system resembles a two-step toll with homogeneous preferences, one step for each group. Similar to the homogeneous case, the step tolling scheme with heterogeneous groups aims to determine the toll window $\left[t_{g}^{+}, t_{g}^{-}\right]$(i.e., the period during which the toll applies) and the toll level $\rho_{g}$ for each group $g(g=1,2)$ so as to maximize the queuing removal (or the total toll revenue). Apparently, both ends of the toll window $\left[t_{g}^{+}, t_{g}^{-}\right]$for group $g$ may be inscribed in its time-varying toll curve or one end of the window is on the time-varying toll curve, whereas the other end is on the boundary $t=t_{12}$. This leads to a total of four possible scenarios, as shown in Fig. 12. The step tolling models for the four scenarios are presented as follows, respectively.

(1) Scenario I: when $t_{2}^{-} \leq t_{12} \leq t_{1}^{+}$, both ends of the step toll window for each group are inscribed in the optimal time-varying toll curve, as shown in Fig. 12a. The step tolling scheme is to determine $\rho_{g}, t_{g}^{+}$and $t_{g}^{-}(g=1,2)$ to maximize the total queuing removal subject to $\rho_{g}=\tau\left(t_{g}^{+}\right)=\tau\left(t_{g}^{-}\right)$and $t_{g}^{+}=2 t_{g}^{* *}-t_{g}^{-}, \quad g=1,2$, expressed as

$$
\begin{array}{ll}
\max & \Lambda_{I}\left(\rho_{1}, t_{1}^{+}, t_{1}^{-}, \rho_{2}, t_{2}^{+}, t_{2}^{-}\right)=\left(t_{2}^{-}-t_{2}^{+}\right) \rho_{2} s+\left(t_{1}^{-}-t_{1}^{+}\right) \rho_{1} s, \\
\text { s.t. }\left\{\begin{array}{l}
\rho_{1}=\tau_{1}\left(t_{1}^{+}\right)=\tau_{1}\left(t_{1}^{-}\right), \\
\rho_{2}=\tau_{2}\left(t_{2}^{+}\right)=\tau_{2}\left(t_{2}^{-}\right), \\
t_{1}^{+}=2 t_{1}^{* *}-t_{1}^{-} \\
t_{2}^{+}=2 t_{2}^{* *}-t_{2}^{-},
\end{array}\right.
\end{array}
$$

where the subscript in $\Lambda$ represents the number of the model scenario.

From the first-order optimality condition of model (66)-(67), one can easily derive the optimal solutions of $\rho_{g}, t_{g}^{+}$and $t_{g}^{-}$as follows. 


$$
\left\{\begin{array} { l } 
{ t _ { 1 } ^ { + } = t _ { 1 } ^ { * * } - \frac { \sqrt { 3 } } { 3 } ( t _ { q ^ { \prime } } - t _ { 1 } ^ { * * } ) , } \\
{ t _ { 1 } ^ { - } = t _ { 1 } ^ { * * } + \frac { \sqrt { 3 } } { 3 } ( t _ { q ^ { \prime } } - t _ { 1 } ^ { * * } ) , }
\end{array} \quad \left\{\begin{array} { l } 
{ t _ { 2 } ^ { + } = t _ { 2 } ^ { * * } - \frac { \sqrt { 3 } } { 3 } ( t _ { 2 } ^ { * * } - t _ { q } ) , } \\
{ t _ { 2 } ^ { - } = t _ { 2 } ^ { * * } + \frac { \sqrt { 3 } } { 3 } ( t _ { 2 } ^ { * * } - t _ { q } ) , }
\end{array} \text { and } \left\{\begin{array}{l}
\rho_{1}=\frac{1}{3}\left(\lambda_{1}-\delta\right)\left(t_{q^{\prime}}-t_{1}^{* *}\right)^{2}, \\
\rho_{2}=\frac{1}{3}\left(\lambda_{2}-\delta\right)\left(t_{2}^{* *}-t_{q}\right)^{2} .
\end{array}\right.\right.\right.
$$

By Eq. (68), the total eliminated queuing delay cost, $\Lambda_{I}$, for Scenario I can be given by

$$
\Lambda_{I}=\left(t_{2}^{-}-t_{2}^{+}\right) \rho_{2} s+\left(t_{1}^{-}-t_{1}^{+}\right) \rho_{1} s=\frac{2 \sqrt{3}}{9} s\left(\left(\lambda_{2}-\delta\right)\left(t_{2}^{* *}-t_{q}\right)^{3}+\left(\lambda_{1}-\delta\right)\left(t_{q^{\prime}}-t_{1}^{* *}\right)^{3}\right) .
$$

In addition, according to $t_{2}^{-}$and $t_{1}^{+}$in Eq. (68) and Eq. (59), the condition, $t_{2}^{-} \leq t_{12} \leq t_{1}^{+}$, leading to Scenario I can be further written as

$t_{2}^{* *}+\frac{\sqrt{3}-1}{2} \frac{N_{2}}{s} \leq t_{12} \leq t_{1}^{* *}-\frac{\sqrt{3}-1}{2} \frac{N_{1}}{s}$, or $\frac{\xi-\mu_{2}}{\lambda_{2}-\delta}+\frac{\sqrt{3}-1}{2} \frac{N_{2}}{s} \leq t_{12} \leq \frac{\xi-\mu_{1}}{\lambda_{1}-\delta}-\frac{\sqrt{3}-1}{2} \frac{N_{1}}{s}$.

(2) Scenario II: when $t_{12} \leq \min \left(t_{1}^{+}, t_{2}^{-}\right)$or equivalently $t_{12} \leq \min \left(\frac{\xi-\mu_{1}}{\lambda_{1}-\delta}-\frac{\sqrt{3}-1}{2} \frac{N_{1}}{s}, \frac{\xi-\mu_{2}}{\lambda_{2}-\delta}+\frac{\sqrt{3}-1}{2} \frac{N_{2}}{s}\right)$, one end (i.e., $t_{2}^{+}$) of the step toll window $\left[t_{2}^{+}, t_{2}^{-}\right]$for the high-income group (i.e., group 2) links the optimal time-varying toll curve and the other end links the boundary $t=t_{12}$. However, both ends of the step toll window $\left[t_{1}^{+}, t_{1}^{-}\right]$for the low-income group (i.e., group 1) are inscribed in the optimal time-varying toll curve, as indicated in Fig. 12b. The model for optimizing $\rho_{1}, \rho_{2}, t_{1}^{+}, t_{1}^{-}$, and $t_{2}^{+}$is as below.

$$
\begin{array}{ll}
\max & \Lambda_{I I}\left(\rho_{1}, t_{1}^{+}, t_{1}^{-}, \rho_{2}, t_{2}^{+}\right)=\left(t_{12}-t_{2}^{+}\right) \rho_{2} s+\left(t_{1}^{-}-t_{1}^{+}\right) \rho_{1} s, \\
\text { s.t. }\left\{\begin{array}{l}
\rho_{1}=\tau_{1}\left(t_{1}^{+}\right)=\tau_{1}\left(t_{1}^{-}\right), \\
\rho_{2}=\tau_{2}\left(t_{2}^{+}\right), \\
t_{1}^{+}=2 t_{1}^{* *}-t_{1}^{-} .
\end{array}\right.
\end{array}
$$

The resultant optimal solutions for $\rho_{1}, \rho_{2}, t_{1}^{+}, t_{1}^{-}$, and $t_{2}^{+}$are 


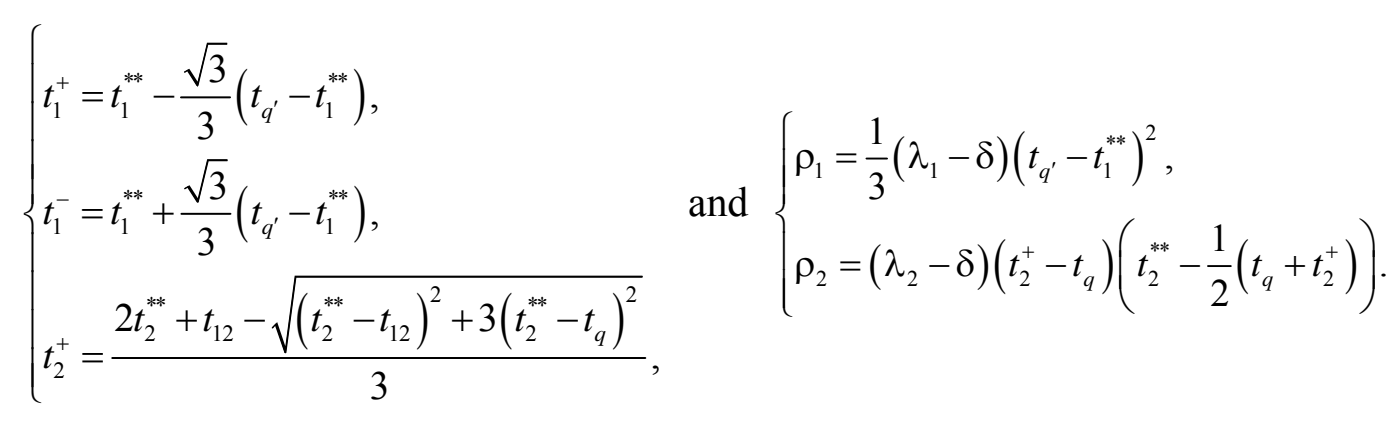

The total eliminated queuing delay cost, $\Lambda_{I I}$, for Scenario II is thus given by

$$
\Lambda_{I I}=\frac{2 \sqrt{3}}{9} s\left(\lambda_{1}-\delta\right)\left(t_{q^{\prime}}-t_{1}^{* *}\right)^{3}+s\left(\lambda_{2}-\delta\right)\left(t_{12}-t_{2}^{+}\right)\left(t_{2}^{+}-t_{q}\right)\left(t_{2}^{* *}-\frac{1}{2}\left(t_{q}+t_{2}^{+}\right)\right) .
$$

(3) Scenario III: when $t_{12} \geq \max \left(t_{1}^{+}, t_{2}^{-}\right)$or equivalently $t_{12} \geq \max \left(\frac{\xi-\mu_{1}}{\lambda_{1}-\delta}-\frac{\sqrt{3}-1}{2} \frac{N_{1}}{s}, \frac{\xi-\mu_{2}}{\lambda_{2}-\delta}+\frac{\sqrt{3}-1}{2} \frac{N_{2}}{s}\right)$, both ends of the step toll window $\left[t_{2}^{+}, t_{2}^{-}\right]$for group 2 are inscribed in the optimal time-varying toll curve. However, one end (i.e., $t_{1}^{-}$) of the step toll window $\left[t_{1}^{+}, t_{1}^{-}\right]$for group 1 is on the time-varying toll curve and the other end (i.e., $t_{1}^{+}$) is on the boundary $t=t_{12}$, as shown in Fig. 12c. The model for determining $\rho_{1}, \rho_{2}, t_{1}^{-}, t_{2}^{+}$, and $t_{2}^{-}$is as below.

$$
\begin{array}{ll}
\max & \Lambda_{I I I}\left(\rho_{1}, t_{1}^{-}, \rho_{2}, t_{2}^{+}, t_{2}^{-}\right)=\left(t_{2}^{-}-t_{2}^{+}\right) \rho_{2} s+\left(t_{1}^{-}-t_{12}\right) \rho_{1} s, \\
\text { s.t. }\left\{\begin{array}{l}
\rho_{1}=\tau_{1}\left(t_{1}^{-}\right), \\
\rho_{2}=\tau_{2}\left(t_{2}^{+}\right)=\tau_{2}\left(t_{2}^{-}\right), \\
t_{2}^{+}=2 t_{2}^{* *}-t_{2}^{-} .
\end{array}\right.
\end{array}
$$

The optimal solutions of model (75)-(76) are given as follows.

$$
\left\{\begin{array}{l}
t_{1}^{-}=\frac{2 t_{1}^{* *}+t_{12}+\sqrt{\left(t_{1}^{* *}-t_{12}\right)^{2}+3\left(t_{1}^{* *}-t_{q^{\prime}}\right)^{2}}}{3} \\
t_{2}^{+}=t_{2}^{* *}-\frac{\sqrt{3}}{3}\left(t_{2}^{* *}-t_{q}\right), \\
t_{2}^{-}=t_{2}^{* *}+\frac{\sqrt{3}}{3}\left(t_{2}^{* *}-t_{q}\right)
\end{array}\right.
$$$$
\text { and }\left\{\begin{array}{l}
\rho_{1}=\left(\lambda_{1}-\delta\right)\left(t_{q^{\prime}}-t_{1}^{-}\right)\left(\frac{1}{2}\left(t_{q^{\prime}}+t_{1}^{-}\right)-t_{1}^{* *}\right), \\
\rho_{2}=\frac{1}{3}\left(\lambda_{2}-\delta\right)\left(t_{2}^{* *}-t_{q}\right)^{2}
\end{array}\right.
$$

The resultant total eliminated queuing delay cost, $\Lambda_{\text {III }}$, for Scenario III is 


$$
\Lambda_{I I I}=s\left(\lambda_{1}-\delta\right)\left(t_{1}^{-}-t_{12}\right)\left(t_{q^{\prime}}-t_{1}^{-}\right)\left(\frac{1}{2}\left(t_{q^{\prime}}+t_{1}^{-}\right)-t_{1}^{* *}\right)+\frac{2 \sqrt{3}}{9} s\left(t_{2}^{* *}-t_{q}\right)^{3}\left(\lambda_{2}-\delta\right) .
$$

(4) Scenario IV: when $t_{1}^{+} \leq t_{12} \leq t_{2}^{-}$or equivalently $\frac{\xi-\mu_{1}}{\lambda_{1}-\delta}-\frac{\sqrt{3}-1}{2} \frac{N_{1}}{\mathrm{~s}} \leq t_{12} \leq \frac{\xi-\mu_{2}}{\lambda_{2}-\delta}+\frac{\sqrt{3}-1}{2} \frac{N_{2}}{\mathrm{~s}}$, one end of the step toll window for each group is on the optimal time-varying toll curve and the other end is on the boundary $t=t_{12}$, as shown in Fig. 12d. The queuing removal maximization problem for determining $\rho_{1}, \rho_{2}, t_{1}^{-}$and $t_{2}^{+}$is represented as

$$
\begin{array}{ll}
\max & \Lambda_{I V}\left(\rho_{1}, t_{1}^{-}, \rho_{2}, t_{2}^{+}\right)=\left(t_{1}^{-}-t_{12}\right) \rho_{1} s+\left(t_{12}-t_{2}^{+}\right) \rho_{2} s, \\
\text { s.t. }\left\{\begin{array}{l}
\rho_{1}=\tau_{1}\left(t_{1}^{-}\right), \\
\rho_{2}=\tau_{2}\left(t_{2}^{+}\right) .
\end{array}\right.
\end{array}
$$

The resultant optimal solutions and total eliminated queuing delay cost for Scenario IV are

$$
\begin{aligned}
& \left\{\begin{array} { l } 
{ t _ { 1 } ^ { - } = \frac { 2 t _ { 1 } ^ { * * } + t _ { 1 2 } + \sqrt { ( t _ { 1 } ^ { * * } - t _ { 1 2 } ) ^ { 2 } + 3 ( t _ { 1 } ^ { * * } - t _ { q ^ { \prime } } ) ^ { 2 } } } { 3 } , } \\
{ t _ { 2 } ^ { + } = \frac { 2 t _ { 2 } ^ { * * } + t _ { 1 2 } - \sqrt { ( t _ { 2 } ^ { * * } - t _ { 1 2 } ) ^ { 2 } + 3 ( t _ { 2 } ^ { * * } - t _ { q } ) ^ { 2 } } } { 3 } , }
\end{array} \text { and } \left\{\begin{array}{l}
\rho_{1}=\left(\lambda_{1}-\delta\right)\left(t_{q^{\prime}}-t_{1}^{-}\right)\left(\frac{1}{2}\left(t_{q^{\prime}}+t_{1}^{-}\right)-t_{1}^{* *}\right), \\
\rho_{2}=\left(\lambda_{2}-\delta\right)\left(t_{2}^{+}-t_{q}\right)\left(t_{2}^{* *}-\frac{1}{2}\left(t_{q}+t_{2}^{+}\right)\right) .
\end{array}\right.\right. \\
& \Lambda_{I V}=s\left(\lambda_{1}-\delta\right)\left(t_{1}^{-}-t_{12}\right)\left(t_{q^{\prime}}-t_{1}^{-}\right)\left(\frac{1}{2}\left(t_{q^{\prime}}+t_{1}^{-}\right)-t_{1}^{* *}\right)+s\left(\lambda_{2}-\delta\right)\left(t_{12}-t_{2}^{+}\right)\left(t_{2}^{+}-t_{q}\right)\left(t_{2}^{* *}-\frac{1}{2}\left(t_{q}+t_{2}^{+}\right)\right) .
\end{aligned}
$$

For the convenience of the readers, the optimal solutions for the step toll levels $\rho_{g}$ and the charging time points $t_{g}^{+}$and $t_{g}^{-}$and the total eliminated queuing delay cost $\Lambda$ under different scenarios are further summarized in Table 2.

In order to assess the efficacy of a step toll scheme, one can calculate the queuing removal rate, which is the proportion of the removed queuing delay cost $\Lambda$ to the total queuing delay cost under the no-toll equilibrium $\Phi^{\mathrm{NT}}$. The total queuing delay cost, $\Phi^{\mathrm{NT}}$, is equal to the total toll revenue of all commuters in groups 1 and 2, defined as

$$
\begin{aligned}
\Phi^{\mathrm{NT}} & =\int_{t_{q}}^{t_{12}} \tau_{2}(t) s d t+\int_{t_{12}}^{t_{q^{\prime}}} \tau_{1}(t) s d t \\
& =\int_{t_{q}}^{t_{12}}\left(\frac{1}{2}\left(\lambda_{2}-\delta\right)\left(t_{q}^{2}-t^{2}\right)-\left(\xi-\mu_{2}\right)\left(t_{q}-t\right)\right) s d t+\int_{t_{12}}^{t_{q^{\prime}}}\left(\frac{1}{2}\left(\lambda_{1}-\delta\right)\left(t_{q^{\prime}}^{2}-t^{2}\right)-\left(\xi-\mu_{1}\right)\left(t_{q^{\prime}}-t\right)\right) s d t \\
& =\frac{1}{6} s\left(\lambda_{2}-\delta\right)\left(t_{12}-t_{q}\right)^{2}\left(3 t_{2}^{* *}-2 t_{q}-t_{12}\right)+\frac{1}{6} s\left(\lambda_{1}-\delta\right)\left(t_{q^{\prime}}-t_{12}\right)^{2}\left(2 t_{q^{\prime}}+t_{12}-3 t_{1}^{* *}\right) .
\end{aligned}
$$


From Eqs. (69), (74), (78), (82) and (83), the queuing removal rate $(Q R R)$ for all scenarios can be calculated by $\frac{\Lambda}{\Phi^{\mathrm{NT}}}$. In contrast to the $Q R R$ of the single-step tolling scheme with the homogeneous preferences (its $Q R R$ value is $\sqrt{3} / 3$, see Proposition 5), we obtain an important property about the efficacy of a step toll scheme. Its proof is provided in Appendix C.

Proposition 9. When the preference heterogeneity is considered in the activity-based bottleneck model with linear marginal utilities, the queuing removal rate from the optimal single-step toll scheme would exceed $\frac{\sqrt{3}}{3}$. This implies that the bottleneck model with homogeneous preferences would underestimate the efficacy of a step toll scheme in terms of the queuing removal rate, compared to the counterpart with heterogeneous preferences.

Proposition 9 shows that the heterogeneity of commuters plays a significant role in evaluating the efficacy of congestion tolling scheme, and the assumption of commuters' homogeneity can lead to a biased estimation of the efficacy. Therefore, it is important to incorporate the commuters' preference heterogeneity in the congestion tolling models.

\subsection{Numerical illustration}

In this section, an example is used to illustrate the properties of the proposed step tolling models with heterogeneous groups. Herein, we only look at $\mu$ heterogeneity because (i) both $\mu$ and $\lambda$ have similar effects on marginal work utility in terms of Eq. (57); and (ii) a closed-form equilibrium solution exists for the $\mu$ heterogeneity, but not for the $\lambda$ heterogeneity.

Under the $\mu$ heterogeneity, the parameters $\xi, \delta$ and $\lambda$ are assumed to be identical across groups, i.e., $\xi_{1}=\xi_{2}, \delta_{1}=\delta_{2}$ and $\lambda_{1}=\lambda_{2}$. According to Eqs. (59)-(61), one can derive the boundary time $t_{12}$ between group 1's and 2's departures and the associated queuing delay time $T\left(t_{12}\right)$ as 


$$
\left\{\begin{array}{l}
T\left(t_{12}\right)=\frac{\lambda-\delta}{\mu_{1}-\mu_{2}} \frac{N}{s} t_{12}+\eta \\
t_{12}=\frac{-\omega_{2}-\sqrt{\omega_{2}^{2}-4 \omega_{1} \omega_{3}}}{2 \omega_{1}},
\end{array}\right.
$$

where

$$
\left\{\begin{array}{l}
\eta=\frac{\lambda-\delta}{\mu_{1}-\mu_{2}} \frac{N\left(N_{1}-N_{2}\right)}{2 s^{2}}-\frac{\xi-\mu_{1}}{\mu_{1}-\mu_{2}} \frac{N_{1}}{s}-\frac{\xi-\mu_{2}}{\mu_{1}-\mu_{2}} \frac{N_{2}}{s}, \\
\omega_{1}=\frac{\lambda(\lambda-\delta)}{\mu_{1}-\mu_{2}} \frac{N}{s}+\frac{\lambda}{2}\left(\frac{\lambda-\delta}{\mu_{1}-\mu_{2}}\right)^{2} \frac{N^{2}}{s^{2}}, \\
\omega_{2}=\frac{\mu_{1}(\lambda-\delta)}{\mu_{1}-\mu_{2}} \frac{N}{s}+\left(1+\frac{\lambda-\delta}{\mu_{1}-\mu_{2}} \frac{N}{s}\right) \lambda \eta-(\lambda-\delta) \frac{N_{1}}{s}, \\
\omega_{3}=\mu_{1} \eta+\frac{\lambda}{2} \eta^{2}-\frac{1}{2}(\lambda-\delta)\left(\frac{N_{1}}{s}\right)^{2}+\left(\xi-\mu_{1}\right) \frac{N_{1}}{s} .
\end{array}\right.
$$

In the example, the number of commuters, $N_{1}$, in group 1 is assumed to be 4000 , and the capacity $s$ of the bottleneck is 4000 vehicles per hour. Again, the linear marginal utility functions for home and work activities are adopted, with parameters $\xi_{1}=\xi_{2}=57(\$ / \mathrm{h})$, $\delta_{1}=\delta_{2}=-8.86\left(\$ / \mathrm{h}^{2}\right)$ and $\lambda_{1}=\lambda_{2}=25.42\left(\$ / \mathrm{h}^{2}\right) . \mu_{1}$ is assumed to be $\$ 16$ per hour. In the following, we will examine the effects of $\mu_{2}$ and $N_{2}$ on the step tolling system.

Fig. 13 shows the effects of $\mu_{2}$ on the step tolling model adopted and the optimal step toll scheme for given $N_{2}=4000$. It can be seen that as $\mu_{2}$ increases from $\$ 25$ to $\$ 50$ per hour, the step tolling model adopted changes in the order of IV, II, and I, and for a given group the step toll in turn decreases. Specifically, the step toll decreases from $\$ 9.8$ to $\$ 2.9$ for group 2 and from $\$ 8.8$ to $\$ 2.8$ for group 1, respectively. This is because the increase in $\mu_{2}$ leads the isocost toll curves for groups 1 and 2 to be gradually separated, thus implying a decreased interaction between the members of groups. Particularly, as $\mu_{2}=25(\$ / \mathrm{h})$, nearly half of the isocost toll curve for each group is overlapped. As a result, the queuing removal rate $(57.97 \%)$ from the step tolls under the heterogeneous preferences is close to that under the homogeneous preferences. As $\mu_{2}$ is doubled (i.e. $\mu_{2}=50(\$ / \mathrm{h})$ ), the isocost toll curves for the two groups are almost completely separated. Thus, the resultant queuing removal rate $(57.74 \%)$ under the heterogeneous preferences is almost equal to that under the homogeneous 
preferences. It should be pointed out that as $\mu_{2}$ is very close to $16(\$ / \mathrm{h})$ (i.e., the value of its lower bound $\mu_{1}$ ), the isocost toll curves for the two groups become a continuous and symmetric parabolic curve and the single-step tolls for the two groups become the same, as shown in Fig. 7. This is because the right half of the isocost toll curve of group 2 exactly overlaps with the left half of the isocost toll curve of group 1.

Fig. 14 shows the results with $N_{2}=6000$ and $\mu_{2}=40,47$, and $50(\$ / \mathrm{h})$, respectively. It can be noted that the charging duration for group 2 becomes 1.5 hours and all of the queuing removal rates exceed $60 \%$. Similar to the case of $N_{2}=4000$, as $\mu_{2}$ increases from $\$ 40$ to $\$ 50$ per hour, the associated step toll decreases for each group. However, the step tolling models adopted change in the order of IV, III, and I, which is different from the case of $N_{2}=4000$. Comparing Figs. 13 and 14 , we can find that when $\mu_{2}=40(\$ / \mathrm{h})$, the step tolling model adopted is Scenario II for $N_{2}=4000$ but Scenario IV for $N_{2}=6000$. These results show that the travel demand and the marginal work utility have important impacts on the step tolling model adopted and the step toll scheme.

Fig. 15 further shows the changes of the step tolling models adopted and the queuing removal rate with group 2's demand $N_{2}$ and the value of $\mu_{2}$. It can be observed that for $N_{2}=4000$, when $\mu_{2}$ is between 38.43 and $43.04(\$ / h)$, the step tolling model adopted is Scenario II; when $\mu_{2}$ is smaller than $\$ 38.43$ per hour and larger than $\$ 43.04$ per hour, the step tolling models adopted are Scenarios IV and I, respectively. For $N_{2}=6000$, when $\mu_{2}$ takes a value between 46.07 and $48.60(\$ / \mathrm{h})$, Scenario III is adopted. Otherwise, Scenario IV is applied if $\mu_{2}$ is smaller than $\$ 46.07$ per hour, and Scenario I is applied if $\mu_{2}$ exceeds $\$ 48.60$ per hour. It can also be observed that the queuing delay removal rate is always larger than $\sqrt{3} / 3$, and for each of the two demand levels (i.e., $N_{2}=4000$ and 6000), as $\mu_{2}$ increases, the queuing removal rate increases in the region of Scenario IV but decreases in the region of Scenario I. These observations indicate the preference heterogeneity and travel demand level have important impacts on the choice of step toll model.

\section{Conclusion and further studies}


This paper revisits the step tolling issues for a road bottleneck using an activity-based modeling framework. The activity scheduling utilities of commuters at home and at work are assumed to be time-dependent during the day and that commuters make their departure time choices in the morning to maximize their own scheduling utility. The step tolling models with homogeneous and heterogeneous preferences are presented. The properties of the models and the optimal step toll scheme with linear time-varying scheduling utilities are analytically explored.

Some important findings and new insights have been obtained. First, the solution curves of the activity-based bottleneck model with linear marginal utilities are nonlinear, continuous, and differentiable. These curves include the departure rate curve, cumulative departure curve, isocost queuing delay curve, and the social-optimum time-varying congestion toll curve. They are different from the activity-based bottleneck model with constant marginal utilities (i.e., traditional Vickrey's bottleneck model) in which these curves are piecewise constant or linear and non-differentiable. Second, the optimal step toll scheme with linear marginal activity utilities must follow a symmetric/shoulder toll structure. Third, for a given number of toll steps, the efficacy of the optimal step toll scheme is higher in the activity-based bottleneck model with linear marginal utilities than in the activity-based bottleneck model with constant marginal utilities in terms of bottleneck queuing removal rate. Fourth, at the user equilibrium, heterogeneous groups sequentially depart in order of decreasing marginal work utility or increasing marginal home activity utility, and ignoring the preference heterogeneity would underestimate the efficacy of a step toll scheme in terms of the queuing removal rate. These findings have significant implications for guiding the implementation of step toll schemes in practice.

Although this paper provides some new insights into bottleneck tolling issues, some important extensions should be made in further studies.

(1) This paper mainly focused on the step tolling issues in the activity-based bottleneck model with linear marginal activity utilities. Although the linear marginal activity utilities can be regarded as an approximation of any marginal activity utility functions through the first-order Taylor expansion, the properties of the model with the linear marginal utilities may not be applicable to the case with general scheduling preferences. Moreover, empirical evidence on the shape of marginal activity utility functions is still limited. When the marginal activity 
utility functions are unknown, a trial-and-error iterative approach proposed by Li (1999, 2002) and Yang et al. (2004) may be applied, which is left for a future study.

(2) This paper addressed the morning commuting problem, and the evening or day-long commuting problems are not considered. In reality, commuters' morning and evening trip-timing decisions may interact (Zhang et al., 2005; Li et al., 2014). Thus, a day-long activity-based bottleneck model should be developed for the step tolling problem to reveal the differences between the morning and evening commutes in the efficiency gains and the welfare-distributional effects of step tolling.

(3) The proposed activity-based bottleneck models in this paper were deterministic, implying that the effects of uncertainties in bottleneck capacity and travel demand were ignored. However, in reality the uncertainties in the bottleneck capacity and/or the travel demand may affect commuters' activity schedules and departure time choices (Lindsey, 2009; Fosgerau and Lindsey, 2013; Xiao et al., 2015). It is thus necessary to consider the effects of these uncertainties in a future study for further improvement of the robustness of the proposed model.

(4) This study focused on a single road bottleneck, and thus the congestion effects over space cannot be addressed. To do so, an activity-based network traffic assignment model should be developed (e.g., see Lam and Yin, 2001; Lam and Huang, 2002; Li et al., 2010; Fu and Lam, 2014; Liu et al., 2015). In a further study, the proposed models in this paper can be extended to a network context such that the network congestion effects can be explicitly taken into account.

(5) The modeling approach for the optimal step toll scheme in this paper was based on the Laih model (1994), and thus the braking behavior of drivers to avoid the toll payment cannot be incorporated. Recent studies of Lindsey et al. (2012) and Xiao et al. (2012) showed that drivers' braking behavior can affect the design of the step tolling system. Therefore, there is a need to incorporate braking behavior in the activity-based step tolling model in a future study.

\section{Acknowledgments}


We are grateful to Professor Robin Lindsey of University of British Columbia and three anonymous referees for their helpful comments and suggestions on an earlier version of the paper. The work described in this paper was jointly supported by grants from the National Natural Science Foundation of China (71525003, 71222107), the Major Program of National Social Science Foundation of China (13\&ZD175), Huazhong University of Science and Technology (5001300001), the National Basic Research Program of China (973 Program) (2012CB725400), and the Research Grants Council of the Hong Kong Special Administrative Region, China (PolyU 5181/13E, HKU 17208614).

\section{References}

Arnott, R., de Palma, A., Lindsey, R., 1988. Schedule delay and departure time decisions with heterogeneous commuters. Transportation Research Record 1197, 56-57.

Arnott, R., de Palma, A., Lindsey, R., 1990. Economics of a bottleneck. Journal of Urban Economics 27(1), 111-130.

Arnott, R., de Palma, A., Lindsey, R., 1994. The welfare effects of congestion tolls with heterogeneous commuters. Journal of Transport Economics and Policy 28(2), 139-161.

Arnott, R., de Palma, A., Lindsey, R., 1998. Recent developments in the bottleneck model. In: Button, K.J., Verhoef, E.T. (eds.), Road Pricing, Traffic Congestion and the Environment: Issues of Efficiency and Social Feasibility, p.79-110. Aldershot: Edward Elgar.

Börjesson, M., Eliasson, J., Franklin, J., 2012. Valuations of travel time variability in scheduling versus mean-variance models. Transportation Research Part B 46(7), 855-873.

Chen, H., Nie, Y., Yin, Y., 2015. Optimal multi-step toll design under general user heterogeneity. Transportation Research Part B 81, 775-793.

Cohen, Y., 1987. Commuter welfare under peak-period congestion tolls: who gains and who loses? International Journal of Transport Economics 14(3), 239-266.

Engelson, L., Fosgerau, M., 2011. Additive measures of travel time variability. Transportation Research Part B 45(10), 1560-1571.

Ettema, D., Timmermans, H.J.P., 1997. Activity-Based Approaches to Travel Analysis. Pergamon, Oxford.

Ettema, D., Timmermans, H.J.P., 2003. Modeling departure time choice in the context of activity scheduling behaviour. Transportation Research Record 1831, 39-46.

Fosgerau, M., de Palma, A., 2012. Congestion in a city with a central bottleneck. Journal of 
Urban Economics 71(3), 269-277.

Fosgerau, M., Engelson, L., 2011. The value of travel time variance. Transportation Research Part B 45(1), 1-8.

Fosgerau, M., Lindsey, R., 2013. Trip-timing decisions with traffic incidents. Regional Science and Urban Economics 43(5), 764-782.

Fu, X., Lam, W.H.K., 2014. A network equilibrium approach for modeling activity-travel pattern scheduling problems in multi-modal transit networks with uncertainty. Transportation 41(1), 37-55.

Hjorth, K., Börjesson, M., Engelson, L., Fosgerau, M., 2015. Estimating exponential scheduling preferences. Transportation Research Part B 81, 230-251.

Huang H.J., 2000. Fares and tolls in a competitive system with transit and highway: The case with two groups of commuters. Transportation Research Part E 36, 267-284.

Jenelius, E., Mattsson, L.G., Levinson, D., 2011. Traveler delay costs and value of time with trip chains, flexible activity scheduling and information. Transportation Research Part B 45(5), 789-807.

Knockaert, J., Verhoef, E.T., Rouwendal, J., 2016. Bottleneck congestion: differentiating the coarse charge. Transportation Research Part B 83, 59-73.

Laih, C.H., 1994. Queuing at a bottleneck with single and multi-step tolls. Transportation Research Part A 28(3), 197-208.

Laih, C.H., 2004. Effects of the optimal step toll scheme on equilibrium commuter behavior. Applied Economics 36(1), 59-81.

Lam, W.H.K., Huang, H.J., 2002. A combined activity/travel choice model for congested road networks with queues. Transportation 29(1), 5-29.

Lam, W.H.K., Yin, Y., 2001. An activity-based time-dependent traffic assignment model. Transportation Research Part B 35(6), 549-574.

Li, M.Z.F., 1999. Estimating congestion toll by using traffic count data - Singapore's area licensing scheme. Transportation Research Part E 35, 1-10.

Li, M.Z.F., 2002. The role of speed-flow relationship in congestion pricing implementation with an application to Singapore. Transportation Research Part B 36, 731-754.

Li, Z.C., Lam, W.H.K., Wong, S.C., 2014. Bottleneck model revisited: an activity-based perspective. Transportation Research Part B 68, 262-287.

Li, Z.C., Lam, W.H.K., Wong, S.C., Sumalee, A., 2010. An activity-based approach for scheduling multimodal transit services. Transportation 37(5), 751-774.

Lindsey, R., 2004. Existence, uniqueness, and trip cost function properties of user equilibrium 
in the bottleneck model with multiple user classes. Transportation Science 38(3), 293-314.

Lindsey, R., 2009. Cost recovery from congestion tolls with random capacity and demand. Journal of Urban Economics 66(1), 16-24.

Lindsey, R., van den Berg, V.A.C., Verhoef, E.T., 2012. Step tolling with bottleneck queuing congestion. Journal of Urban Economics 72, 46-59.

Liu, H.X., He, X., Recker, W., 2007. Estimation of time-dependency of values of travel time and its reliability from loop detector data. Transportation Research Part B 41, 448-461.

Liu, P., Liao, F.X., Huang, H.J., Timmermans, H., 2015. Dynamic activity-travel assignment in multi-state supernetworks. Transportation Research Part B 81, 656-671.

Small, K.A., 1982. The scheduling of consumer activities: work trips. American Economic Review 72(3), 467-479.

Small, K.A., 2015. The bottleneck model: an assessment and interpretation. Economics of Transportation 4(1-2), 110-117.

Small, K.A., Winston, C., Yan, J., 2005. Uncovering the distribution of motorists' preferences for travel time and reliability. Econometrica 73(4), 1367-1382.

Tseng, Y.Y., Verhoef, E.T., 2008. Value of time by time of day: a stated-preference study. Transportation Research Part B 42(7-8), 607-618.

van den Berg, V.A.C., 2012 Step-tolling with price-sensitive demand: why more steps in the toll make the consumer better off. Transportation Research Part A 46(10), 1608-1622.

van den Berg, V.A.C., 2014. Coarse tolling with heterogeneous preferences. Transportation Research Part B 64, 1-23.

van den Berg, V.A.C., Verhoef, E.T., 2011a. Winning or losing from dynamic bottleneck congestion pricing? The distributional effects of road pricing with heterogeneity in values of time and schedule delay. Journal of Public Economics 95 (7-8), 983-992.

van den Berg, V.A.C., Verhoef, E.T., 2011b. Congestion tolling in the bottleneck model with heterogeneous values of time. Transportation Research Part B 45 (1), 60-78.

Vickrey, W.S., 1969. Congestion theory and transport investment. American Economic Review 59(2), 251-261.

Vickrey, W.S., 1973. Pricing, metering, and efficiently using urban transportation facilities. Highway Research Record 476, 36-48.

Wu, W.X., Huang, H.J., 2014. Finding anonymous tolls to realize target flow pattern in networks with continuously distributed value of time. Transportation Research Part B 65, $31-46$.

Wu, W.X., Huang, H.J., 2015. An ordinary differential equation formulation of the bottleneck 
model with user heterogeneity. Transportation Research Part B 81, 34-58.

Xiao, F., Qian, Z., Zhang, H.M., 2011. The morning commute problem with coarse toll and nonidentical commuters. Networks and Spatial Economics 11(2), 343-369.

Xiao, F., Shen, W., Zhang, H.M., 2012. The morning commute under flat toll and tactical waiting. Transportation Research Part B 46(10), 1346-1359.

Xiao, L.L., Huang, H.J., Liu, R., 2015. Congestion behavior and tolls in a bottleneck model with stochastic capacity. Transportation Science 49(1), 46-65.

Yang, H., Meng, Q., Lee, D.H., 2004. Trial-and-error implementation of marginal-cost pricing on networks in the absence of demand functions. Transportation Research Part B 38(6), 477-493.

Yao, T., Wei, M.M., Zhang, B., Friesz, T., 2012. Congestion derivatives for a traffic bottleneck with heterogeneous commuters. Transportation Research Part B 46, 1454-1473.

Zhang, X.N., Yang, H., Huang, H.J., Zhang, H.M., 2005. Integrated scheduling of daily work activities and morning-evening commutes with bottleneck congestion. Transportation Research Part A 39(1), 41-60. 


\section{Appendix A: Proof of Proposition 1}

(i) We need to prove that there exists some time $\hat{t} \in\left(t_{q}, t_{q^{\prime}}\right)$ where $t_{q}$ and $t_{q^{\prime}}$ are the departure times from home of the first and last commuters in the morning respectively, such that a queue exists at that time. A contradiction method is adopted here.

Suppose that for any time $\hat{t} \in\left(t_{q}, t_{q^{\prime}}\right)$, there is no queue. We then immediately obtain

$$
C(\hat{t})=\int_{\hat{t}}^{t^{* *}}(h(t)-w(t)) d t .
$$

Note that the first commuter departing from home at time $t_{q}$ faces no queue. From Eq. (14), his or her travel cost is

$$
C\left(t_{q}\right)=\int_{t_{q}}^{t^{* *}} h(t) d t+\int_{t^{* *}}^{t_{q}^{*}} w(t) d t=\int_{t_{q}}^{t^{* *}}(h(t)-w(t)) d t .
$$

Thus, we have

$$
C(\hat{t})-C\left(t_{q}\right)=\int_{t_{q}}^{\hat{t}}(w(t)-h(t)) d t .
$$

Under the assumption that $h(t)$ monotonically decreases and $w(t)$ monotonically increases and that both satisfy $h(t)>w(t)$ for $t<t^{* *}$ and $h(t)<w(t)$ for $t>t^{* *}$, we have

$$
C(\hat{t})-C\left(t_{q}\right)\left\{\begin{array}{l}
<0, \text { if } t_{q}<\hat{t} \leq t^{* *}, \\
>0, \text { if } \hat{t}>t_{q} \geq t^{* *}
\end{array}\right.
$$

This contradicts the equilibrium condition of $C(\hat{t})=C\left(t_{q}\right)$ for any $\hat{t}$. Therefore, there exists a time $\hat{t} \in\left(t_{q}, t_{q^{\prime}}\right)$ such that there is a queue at $\hat{t}$.

(ii) The first-order optimality condition of minimization problem (15) implies

$$
\frac{d C(t)}{d t}=-h(t)+\left(1+\frac{d T(t)}{d t}\right) w(t+T(t))=0 .
$$

One thus obtains

$$
\frac{d T(t)}{d t}=\frac{h(t)}{w(t+T(t))}-1
$$

Consequently, the second-order derivative of the queuing delay $T(t)$ is

$$
\frac{d^{2} T(t)}{d t^{2}}=\frac{1}{w(t+T(t))}\left(\frac{d h(\cdot)}{d t}-\left(1+\frac{d T(t)}{d t}\right)^{2} \frac{d w(\cdot)}{d t}\right) .
$$


Because $h(t)$ monotonically decreases and $w(t)$ monotonically increases, $\frac{d h(\cdot)}{d t}<0$ and $\frac{d w(\cdot)}{d t}>0$ hold. In addition, the marginal work activity utility $w(t)$ is positive. Consequently, $\frac{d^{2} T(t)}{d t^{2}}<0$ in terms of Eq. (A.7), implying that the isocost queuing delay curve $T(t)$ is concave. This completes the proof.

\section{Appendix B: Proof of Proposition 6}

The mathematical induction method is adopted. First, we prove that Proposition 6 is true for the two-step toll scheme (i.e., $m=2$ ). For doing so, a contradiction approach is used. Suppose that the optimal two-step toll scheme with the linear marginal activity utilities is not a symmetric (or shoulder) structure with regard to $t^{* *}$, namely $t^{* *}-t_{2}^{+} \neq t_{2}^{-}-t^{* *}$ (or $\left.t_{2}^{+} \neq 2 t^{* *}-t_{2}^{-}\right)$and $\tau\left(t_{2}^{+}\right) \neq \tau\left(t_{2}^{-}\right)$. For ease of presentation, we denote $\tau\left(t_{2}^{+}\right)$as $\rho_{2}$ and $\tau\left(t_{2}^{-}\right)$as $\rho_{2}^{\prime}$, i.e., $\rho_{2}=\tau\left(t_{2}^{+}\right)$and $\rho_{2}^{\prime}=\tau\left(t_{2}^{-}\right)$. The two-step toll scheme aims to determine $\rho_{1}, \rho_{2}, \rho_{2}^{\prime}, t_{1}^{+}, t_{1}^{-}, t_{2}^{+}$, and $t_{2}^{-}$(cf. Fig. 9) to maximize the queuing removal (or the total toll revenue), which is the sum of the areas of the rectangles inscribed in the optimal time-varying toll curve, expressed as

$$
\begin{array}{ll}
\max & \Lambda_{2}\left(\rho_{1}, \rho_{2}, \rho_{2}^{\prime}, t_{1}^{+}, t_{1}^{-}, t_{2}^{+}, t_{2}^{-}\right)=\rho_{1} s\left(t_{1}^{-}-t_{1}^{+}\right)+\rho_{2} s\left(t_{1}^{+}-t_{2}^{+}\right)+\rho_{2}^{\prime} s\left(t_{2}^{-}-t_{1}^{-}\right), \\
\text {s.t. }\left\{\begin{array}{l}
\rho_{1}=\tau\left(t_{1}^{+}\right)=\tau\left(t_{1}^{-}\right), \\
\rho_{2}=\tau\left(t_{2}^{+}\right), \\
\rho_{2}^{\prime}=\tau\left(t_{2}^{-}\right), \\
t_{1}^{+}=2 t^{* *}-t_{1}^{-}, \\
t_{2}^{+} \neq 2 t^{* *}-t_{2}^{-} .
\end{array}\right.
\end{array}
$$

Substituting Eqs. (36) and (B.2) into Eq. (B.1), we obtain

$\max \Lambda_{2}\left(t_{1}^{-}, t_{2}^{+}, t_{2}^{-}\right)=2 s\left(t^{* *}-t_{1}^{-}\right)\left(\frac{1}{2}(\lambda-\delta)\left(t_{1}^{-}\right)^{2}-(\xi-\mu) t_{1}^{-}\right)+s\left(t_{1}^{-}+t_{2}^{+}-2 t^{* *}\right)\left(\frac{1}{2}(\lambda-\delta)\left(t_{2}^{+}\right)^{2}-(\xi-\mu) t_{2}^{+}\right)$ 


$$
+s\left(t_{1}^{-}-t_{2}^{-}\right)\left(\frac{1}{2}(\lambda-\delta)\left(t_{2}^{-}\right)^{2}-(\xi-\mu) t_{2}^{-}\right)+s\left(t_{2}^{-}-t_{2}^{+}\right)\left(\frac{N^{2}}{8 s^{2}}(\lambda-\delta)-\frac{(\xi-\mu)^{2}}{2(\lambda-\delta)}\right) .
$$

From the first-order optimality condition of the maximization problem (B.3), we have

$$
\left\{\begin{array}{l}
\frac{\partial \Lambda_{2}}{\partial t_{1}^{-}}=s(\lambda-\delta)\left(2 t_{1}^{-} t^{* *}-3\left(t_{1}^{-}\right)^{2}+\frac{1}{2}\left(t_{2}^{+}\right)^{2}+\frac{1}{2}\left(t_{2}^{-}\right)^{2}\right)-s(\xi-\mu)\left(2 t^{* *}-4 t_{1}^{-}+t_{2}^{+}+t_{2}^{-}\right)=0, \\
\frac{\partial \Lambda_{2}}{\partial t_{2}^{+}}=s(\lambda-\delta)\left(-2 t_{2}^{+} t^{* *}+t_{1}^{-} t_{2}^{+}+\frac{3}{2}\left(t_{2}^{+}\right)^{2}-\frac{N^{2}}{8 s^{2}}\right)-s(\xi-\mu)\left(-2 t^{* *}+2 t_{2}^{+}+t_{1}^{-}-\frac{\xi-\mu}{2(\lambda-\delta)}\right)=0, \\
\frac{\partial \Lambda_{2}}{\partial t_{2}^{-}}=s(\lambda-\delta)\left(-\frac{3}{2}\left(t_{2}^{-}\right)^{2}+t_{1}^{-} t_{2}^{-}+\frac{N^{2}}{8 s^{2}}\right)-s(\xi-\mu)\left(t_{1}^{-}-2 t_{2}^{-}+\frac{\xi-\mu}{2(\lambda-\delta)}\right)=0 .
\end{array}\right.
$$

Summing the second and third equations in Eq. (B.4) and substituting $t^{* *}=\frac{\xi-\mu}{\lambda-\delta}$, we obtain

$$
\left(t_{2}^{+}+t_{2}^{-}-2 t^{* *}\right)\left(t_{1}^{-}+\frac{3}{2} t_{2}^{+}-\frac{3}{2} t_{2}^{-}-t^{* *}\right)=0 .
$$

We thus have

$$
t_{2}^{+}=2 t^{* *}-t_{2}^{-} .
$$

This contradicts the assumption of $t_{2}^{+} \neq 2 t^{* *}-t_{2}^{-}$. Therefore, the optimal two-step toll scheme with the linear marginal activity utilities follows a symmetric toll structure.

We now prove that Proposition 6 is true for the $(m+1)$-step toll scheme, assuming the truth of the statement for the $m$-step toll scheme. This means that the optimal $m$-step toll scheme with the linear marginal activity utilities is symmetric, i.e., $t^{* *}-t_{i}^{+}=t_{i}^{-}-t^{* *}$ (or $t_{i}^{+}=2 t^{* *}-t_{i}^{-}$) and $\rho_{i}=\rho_{i}^{\prime}(i=1,2, \ldots, m)$. Again, a contradiction approach is adopted. Suppose that the $(m+1)$ th-step toll does not follow a symmetric toll structure, i.e., $t_{m+1}^{+} \neq 2 t^{* *}-t_{m+1}^{-}$and $\rho_{m+1} \neq \rho_{m+1}^{\prime}$. The $(m+1)$-step toll scheme aims to determine tolling time points $t_{i}^{+}$and $t_{i}^{-}(i=1,2, \ldots, m+1)$ and toll levels $\rho_{i}$ and $\rho_{i}^{\prime}(i=1,2, \ldots, m+1)$ to maximize the queuing removal (or the total toll revenue), subject to $t_{i}^{+}=-t_{i}^{-}, \rho_{i}=\rho_{i}^{\prime} \quad(i=1,2, \ldots, m)$, $t_{m+1}^{+} \neq 2 t^{* *}-t_{m+1}^{-}$and $\rho_{m+1} \neq \rho_{m+1}^{\prime}$. The resultant maximization problem is as below $\max \Lambda_{m+1}\left(\rho_{1}, \ldots, \rho_{m+1}, t_{1}^{+}, t_{1}^{-}, \ldots, t_{m+1}^{+}, t_{m+1}^{-}\right)=\rho_{1} s\left(t_{1}^{-}-t_{1}^{+}\right)+2 \rho_{2} s\left(t_{2}^{-}-t_{1}^{-}\right)+\cdots+2 \rho_{i} s\left(t_{i}^{-}-t_{i-1}^{-}\right)+\cdots+2 \rho_{m} s\left(t_{m}^{-}-t_{m-1}^{-}\right)$

$$
+\rho_{m+1} s\left(t_{m}^{+}-t_{m+1}^{+}\right)+\rho_{m+1}^{\prime} s\left(t_{m+1}^{-}-t_{m}^{-}\right),
$$


s.t. $\left\{\begin{array}{l}\rho_{i}=\tau\left(t_{i}^{+}\right)=\tau\left(t_{i}^{-}\right), i=1,2, \ldots, m, \\ t_{i}^{+}=2 t^{* *}-t_{i}^{-}, i=1,2, \ldots, m, \\ \rho_{m+1}=\tau\left(t_{m+1}^{+}\right), \\ \rho_{m+1}^{\prime}=\tau\left(t_{m+1}^{-}\right), \\ t_{m+1}^{+} \neq 2 t^{* *}-t_{m+1}^{-} .\end{array}\right.$

Substituting Eqs. (36) and (B.8) into (B.7), we obtain

$$
\begin{aligned}
& \max \Lambda_{m+1}\left(\rho_{1}, \ldots, \rho_{m+1}, t_{1}^{-}, \ldots, t_{m}^{-}, t_{m+1}^{+}, t_{m+1}^{-}\right)=2 s\left(t^{* *}-t_{1}^{-}\right)\left(\frac{1}{2}(\lambda-\delta)\left(t_{1}^{-}\right)^{2}-(\xi-\mu) t_{1}^{-}\right) \\
& -\sum_{i=2}^{m} 2 s\left(t_{i}^{-}-t_{i-1}^{-}\right)\left(\frac{1}{2}(\lambda-\delta)\left(t_{i}^{-}\right)^{2}-(\xi-\mu) t_{i}^{-}\right)+s\left(t_{m}^{-}+t_{m+1}^{+}-2 t^{* *}\right)\left(\frac{1}{2}(\lambda-\delta)\left(t_{m+1}^{+}\right)^{2}-(\xi-\mu) t_{m+1}^{+}\right) \\
& +s\left(t_{m}^{-}-t_{m+1}^{-}\right)\left(\frac{1}{2}(\lambda-\delta)\left(t_{m+1}^{-}\right)^{2}-(\xi-\mu) t_{m+1}^{-}\right)+s\left(t_{m+1}^{-}-t_{m+1}^{+}\right)\left(\frac{N^{2}}{8 s^{2}}(\lambda-\delta)-\frac{(\xi-\mu)^{2}}{2(\lambda-\delta)}\right)
\end{aligned}
$$

The first-order optimality condition of maximization problem (B.9) can be derived as follows.

$$
\left\{\begin{array}{l}
\frac{3}{2}\left(t_{1}^{-}\right)^{2}-3 t_{1}^{-} t^{* *}-\frac{1}{2}\left(t_{2}^{-}\right)^{2}+t_{2}^{-} t^{* *}+\left(t^{* *}\right)^{2}=0, \\
3\left(t_{i}^{-}\right)^{2}-2 t_{i-1}^{-} t_{i}^{-}-\left(t_{i+1}^{-}\right)^{2}-2 t^{* *}\left(2 t_{i}^{-}-t_{i-1}^{-}-t_{i+1}^{-}\right)=0, i=2,3, \ldots, m-1, \\
3\left(t_{m}^{-}\right)^{2}-2 t_{m-1}^{-} t_{m}^{-}-\frac{1}{2}\left(t_{m+1}^{+}\right)^{2}-\frac{1}{2}\left(t_{m+1}^{-}\right)^{2}-t^{* *}\left(4 t_{m}^{-}-2 t_{m-1}^{-}-t_{m+1}^{+}-t_{m+1}^{-}\right)=0, \\
\frac{3}{2}\left(t_{m+1}^{+}\right)^{2}-2 t_{m+1}^{+} t^{* *}+t_{m}^{-} t_{m+1}^{+}-\frac{N^{2}}{8 s^{2}}-t^{* *}\left(2 t_{m+1}^{+}-2 t^{* *}+t_{m}^{-}-\frac{\xi-\mu}{2(\lambda-\delta)}\right)=0, \\
-\frac{3}{2}\left(t_{m+1}^{-}\right)^{2}+t_{m}^{-} t_{m+1}^{-}+\frac{N^{2}}{8 s^{2}}-t^{* *}\left(-2 t_{m+1}^{-}+t_{m}^{-}+\frac{\xi-\mu}{2(\lambda-\delta)}\right)=0 .
\end{array}\right.
$$

Summing the last two equations in Eq. (B.10), one obtains

$$
\left(t_{m+1}^{+}+t_{m+1}^{-}-2 t^{* *}\right)\left(t_{m}^{-}+\frac{3}{2} t_{m+1}^{+}-\frac{3}{2} t_{m+1}^{-}-t^{* *}\right)=0 .
$$

Eq. (B.11) implies

$$
t_{m+1}^{+}=2 t^{* *}-t_{m+1}^{-} \text {. }
$$

This contradicts the assumption of $t_{m+1}^{+} \neq 2 t^{* *}-t_{m+1}^{-}$. Consequently, the $(m+1)$ th-step toll follows a symmetric structure, and Proposition 6 is thus true for $(m+1)$-step toll scheme. This completes the proof of this proposition. 


\section{Appendix C: Proof of Proposition 9}

Note that $\mu_{1} \leq \mu_{2}<\xi$ holds if $t^{* *}>0$. The change of $\mu_{2}$ from $\mu_{1}$ to $\xi$ leads to four scenarios of the step toll model, i.e., Scenarios IV, III, II, and I, as formulated in Subsection 5.2.2. When $\mu_{2}$ is enough large (but smaller than $\xi$ ), the isocost toll curves for groups 1 and 2 are fully disjoint. For such an extreme case, model I is applicable and the associated total queuing delay $\Phi^{\mathrm{NT}}$ for all commuters in groups 1 and 2 can be given by

$$
\Phi^{\mathrm{NT}}=\int_{t_{q}}^{2 t_{2}^{*}-t_{q}} \tau_{2}(t) s d t+\int_{2 t_{1}^{\prime \prime}-t_{q^{\prime}}}^{t_{q^{\prime}}} \tau_{1}(t) s d t,
$$

where $2 t_{2}^{* *}-t_{q}$ is the symmetric point of $t_{q}$ with regard to $t_{2}^{* *}$, and $2 t_{1}^{* *}-t_{q^{\prime}}$ is the symmetric point of $t_{q^{\prime}}$ with regard to $t_{1}^{* *}$.

Substituting Eqs. (63) and (65) into (C.1) yields

$$
\Phi^{\mathrm{NT}}=\frac{2}{3} s\left(\left(\lambda_{2}-\delta\right)\left(t_{2}^{* *}-t_{q}\right)^{3}+\left(\lambda_{1}-\delta\right)\left(t_{q^{\prime}}-t_{1}^{* *}\right)^{3}\right) \text {. }
$$

By Eq. (69), the removed queuing delay cost by the step tolling scheme is

$$
\Lambda=\frac{2 \sqrt{3}}{9} s\left(\left(\lambda_{2}-\delta\right)\left(t_{2}^{* *}-t_{q}\right)^{3}+\left(\lambda_{1}-\delta\right)\left(t_{q^{\prime}}-t_{1}^{* *}\right)^{3}\right)
$$

The queuing removal rate $Q R R$ is thus calculated as

$$
Q R R=\frac{\Lambda}{\Phi^{\mathrm{NT}}}=\frac{\sqrt{3}}{3} .
$$

As $\mu_{2}$ gradually decreases, the two isocost curves start to intersect, implying that the queuing removal rate $Q R R$ for each group increases and its value is thus larger than $\frac{\sqrt{3}}{3}$. As $\mu_{2}$ continues to decrease such that $t_{12}$ arrives at $t_{2}^{-}$(or $t_{1}^{+}$), the queuing removal rate $Q R R$ for group 2 (or group 1) decreases. When $\mu_{2}$ decreases to $\mu_{1}$ (i.e., $\mu_{2}=\mu_{1}$ ), $t_{12}$ coincides with both $t_{2}^{* *}$ and $t_{1}^{* *}$. For this extreme case, $t_{12}$ becomes the symmetric axis of the isocost toll curve, which is composed of left half of the isocost toll curve for group 2 and right half of the isocost toll curve for group 1. Accordingly, the resultant total queuing delay is half of $\Phi^{\mathrm{NT}}$ in Eq. (C.2), and the total eliminated queuing delay is also just half of $\Lambda$ in Eq. 
(C.3). The queuing removal rate $Q R R$ thus remains to be $\frac{\sqrt{3}}{3}$. To sum up, as $\mu_{1}<\mu_{2}<\xi$, the queuing removal rate $Q R R$ would exceed $\frac{\sqrt{3}}{3}$, which is the $Q R R$ under the two extreme cases: $\mu_{2}$ is, respectively, infinitely close to its upper bound $\xi$ and its lower bound $\mu_{1}$. This completes this proof of this proposition. 
Table 1 The optimal solutions for single-step and two-step toll schemes for activity-based bottleneck model with linear marginal activity utilities.

\begin{tabular}{|c|c|c|c|}
\hline $\begin{array}{l}\text { Tolling } \\
\text { scheme }\end{array}$ & Step toll $\rho_{i}$ & $\begin{array}{l}\text { Charging duration } \\
\text { for each step }\end{array}$ & $\begin{array}{l}\text { Queuing removal rate } \\
\qquad Q R R=\frac{\Lambda}{\Phi^{\mathrm{NT}}}\end{array}$ \\
\hline Single-step & $\rho_{1}=\frac{2}{3} \tau_{\max }$ & $t_{1}^{-}-t_{1}^{+}=\frac{\sqrt{3}}{3} \frac{N}{s}$ & $\frac{\sqrt{3}}{3} \approx 0.577$ \\
\hline Two-step & $\begin{array}{l}\rho_{1}=\frac{8-2 \sqrt{3}}{9-2 \sqrt{3}} \tau_{\max } \approx 0.819 \tau_{\max } \\
\rho_{2}=\frac{6-2 \sqrt{3}}{9-2 \sqrt{3}} \tau_{\text {max }} \approx 0.458 \tau_{\max }\end{array}$ & $\begin{array}{l}t_{1}^{-}-t_{1}^{+}=\frac{1}{\sqrt{9-2 \sqrt{3}}} \frac{N}{\mathrm{~s}} \approx 0.425 \frac{\mathrm{N}}{\mathrm{s}}, \\
2\left(t_{2}^{-}-t_{1}^{-}\right)=\frac{\sqrt{3}-1}{\sqrt{9-2 \sqrt{3}}} \frac{N}{\mathrm{~s}} \approx 0.311 \frac{\mathrm{N}}{\mathrm{s}}\end{array}$ & $\frac{(3 \sqrt{3}-2) \sqrt{9-2 \sqrt{3}}}{31-12 \sqrt{3}} \approx 0.736$ \\
\hline
\end{tabular}

Note: $\tau_{\max }=\frac{N^{2}}{8 s^{2}}(\lambda-\delta)$ and $\Phi^{\mathrm{NT}}=\frac{N^{3}}{12 s^{2}}(\lambda-\delta)$. 
Table 2 Optimal solutions of step toll schemes for different scenarios.

\begin{tabular}{|c|c|c|c|c|}
\hline Scenario & Condition & Optimal tolling time points & Optimal step tolls & Eliminated queuing delay cost \\
\hline I & $\frac{\xi-\mu_{2}}{\lambda_{2}-\delta}+\frac{\sqrt{3}-1}{2} \frac{N_{2}}{s} \leq t_{12} \leq \frac{\xi-\mu_{1}}{\lambda_{1}-\delta}-\frac{\sqrt{3}-1}{2} \frac{N_{1}}{s}$ & $\left\{\begin{array}{l}t_{1}^{+}=t_{1}^{* *}-\frac{\sqrt{3}}{3}\left(t_{q^{\prime}}-t_{1}^{* *}\right) \\
t_{1}^{-}=t_{1}^{* *}+\frac{\sqrt{3}}{3}\left(t_{q^{\prime}}-t_{1}^{* *}\right) \\
t_{2}^{+}=t_{2}^{* *}-\frac{\sqrt{3}}{3}\left(t_{2}^{* *}-t_{q}\right) \\
t_{2}^{-}=t_{2}^{* *}+\frac{\sqrt{3}}{3}\left(t_{2}^{* *}-t_{q}\right)\end{array}\right.$ & $\left\{\begin{array}{l}\rho_{1}=\frac{1}{3}\left(\lambda_{1}-\delta\right)\left(t_{q^{\prime}}-t_{1}^{* *}\right)^{2} \\
\rho_{2}=\frac{1}{3}\left(\lambda_{2}-\delta\right)\left(t_{2}^{* *}-t_{q}\right)^{2}\end{array}\right.$ & $\Lambda_{I}=\frac{2 \sqrt{3}}{9} s\left(\lambda_{1}-\delta\right)\left(t_{q^{\prime}}-t_{1}^{* *}\right)^{3}+\frac{2 \sqrt{3}}{9} s\left(\lambda_{2}-\delta\right)\left(t_{2}^{* * *}-t_{q}\right)^{3}$ \\
\hline II & $t_{12} \leq \min \left(\frac{\xi-\mu_{1}}{\lambda_{1}-\delta}-\frac{\sqrt{3}-1}{2} \frac{N_{1}}{s}, \frac{\xi-\mu_{2}}{\lambda_{2}-\delta}+\frac{\sqrt{3}-1}{2} \frac{N_{2}}{s}\right)$ & $\left\{\begin{array}{l}t_{1}^{+}=t_{1}^{* *}-\frac{\sqrt{3}}{3}\left(t_{q^{\prime}}-t_{1}^{* *}\right) \\
t_{1}^{-}=t_{1}^{* *}+\frac{\sqrt{3}}{3}\left(t_{q^{\prime}}-t_{1}^{* *}\right) \\
t_{2}^{+}=\frac{2 t_{2}^{* *}+t_{12}-\sqrt{\left(t_{2}^{* *}-t_{12}\right)^{2}+3\left(t_{2}^{* *}-t_{q}\right)^{2}}}{3}\end{array}\right.$ & $\begin{array}{l}\rho_{1}=\frac{1}{3}\left(\lambda_{1}-\delta\right)\left(t_{q^{\prime}}-t_{1}^{* *}\right)^{2} \\
\rho_{2}=\left(\lambda_{2}-\delta\right)\left(t_{2}^{+}-t_{q}\right)\left(t_{2}^{* *}-\frac{1}{2}\left(t_{q}+t_{2}^{+}\right)\right)\end{array}$ & $\begin{aligned} \Lambda_{I I}= & \frac{2 \sqrt{3}}{9} s\left(\lambda_{1}-\delta\right)\left(t_{q^{\prime}}-t_{1}^{* *}\right)^{3} \\
& +s\left(\lambda_{2}-\delta\right)\left(t_{12}-t_{2}^{+}\right)\left(t_{2}^{+}-t_{q}\right)\left(t_{2}^{* *}-\frac{1}{2}\left(t_{q}+t_{2}^{+}\right)\right)\end{aligned}$ \\
\hline III & $t_{12} \geq \max \left(\frac{\xi-\mu_{1}}{\lambda_{1}-\delta}-\frac{\sqrt{3}-1}{2} \frac{N_{1}}{s}, \frac{\xi-\mu_{2}}{\lambda_{2}-\delta}+\frac{\sqrt{3}-1}{2} \frac{N_{2}}{s}\right)$ & $\left\{\begin{array}{l}t_{1}^{-}=\frac{2 t_{1}^{* *}+t_{12}+\sqrt{\left(t_{1}^{* *}-t_{12}\right)^{2}+3\left(t_{1}^{* *}-t_{q^{\prime}}\right)^{2}}}{3} \\
t_{2}^{+}=t_{2}^{* *}-\frac{\sqrt{3}}{3}\left(t_{2}^{* *}-t_{q}\right) \\
t_{2}^{-}=t_{2}^{* *}+\frac{\sqrt{3}}{3}\left(t_{2}^{* *}-t_{q}\right)\end{array}\right.$ & $\begin{array}{l}\rho_{1}=\left(\lambda_{1}-\delta\right)\left(t_{q^{\prime}}-t_{1}^{-}\right)\left(\frac{1}{2}\left(t_{q^{\prime}}+t_{1}^{-}\right)-t_{1}^{* *}\right) \\
\rho_{2}=\frac{1}{3}\left(\lambda_{2}-\delta\right)\left(t_{2}^{* *}-t_{q}\right)^{2}\end{array}$ & $\begin{aligned} \Lambda_{I I I}= & s\left(\lambda_{1}-\delta\right)\left(t_{1}^{-}-t_{12}\right)\left(t_{q^{\prime}}-t_{1}^{-}\right)\left(\frac{1}{2}\left(t_{q^{\prime}}+t_{1}^{-}\right)-t_{1}^{* *}\right) \\
& +\frac{2 \sqrt{3}}{9} s\left(t_{2}^{* *}-t_{q}\right)^{3}\left(\lambda_{2}-\delta\right)\end{aligned}$ \\
\hline IV & $\frac{\xi-\mu_{1}}{\lambda_{1}-\delta}-\frac{\sqrt{3}-1}{2} \frac{N_{1}}{s} \leq t_{12} \leq \frac{\xi-\mu_{2}}{\lambda_{2}-\delta}+\frac{\sqrt{3}-1}{2} \frac{N_{2}}{s}$ & $\left\{\begin{array}{l}t_{1}^{-}=\frac{2 t_{1}^{* *}+t_{12}+\sqrt{\left(t_{1}^{* *}-t_{12}\right)^{2}+3\left(t_{1}^{* *}-t_{q^{\prime}}\right)^{2}}}{3} \\
t_{2}^{+}=\frac{2 t_{2}^{* *}+t_{12}-\sqrt{\left(t_{2}^{* *}-t_{12}\right)^{2}+3\left(t_{2}^{* *}-t_{q}\right)^{2}}}{3}\end{array}\right.$ & $\begin{array}{l}\rho_{1}=\left(\lambda_{1}-\delta\right)\left(t_{q^{\prime}}-t_{1}^{-}\right)\left(\frac{1}{2}\left(t_{q^{\prime}}+t_{1}^{-}\right)-t_{1}^{* *}\right) \\
\rho_{2}=\left(\lambda_{2}-\delta\right)\left(t_{2}^{+}-t_{q}\right)\left(t_{2}^{* * *}-\frac{1}{2}\left(t_{q}+t_{2}^{+}\right)\right)\end{array}$ & $\begin{aligned} \Lambda_{I V}= & s\left(\lambda_{1}-\delta\right)\left(t_{1}^{-}-t_{12}\right)\left(t_{q^{\prime}}-t_{1}^{-}\right)\left(\frac{1}{2}\left(t_{q^{\prime}}+t_{1}^{-}\right)-t_{1}^{* *}\right) \\
& +s\left(\lambda_{2}-\delta\right)\left(t_{12}-t_{2}^{+}\right)\left(t_{2}^{+}-t_{q}\right)\left(t_{2}^{* *}-\frac{1}{2}\left(t_{q}+t_{2}^{+}\right)\right)\end{aligned}$ \\
\hline
\end{tabular}




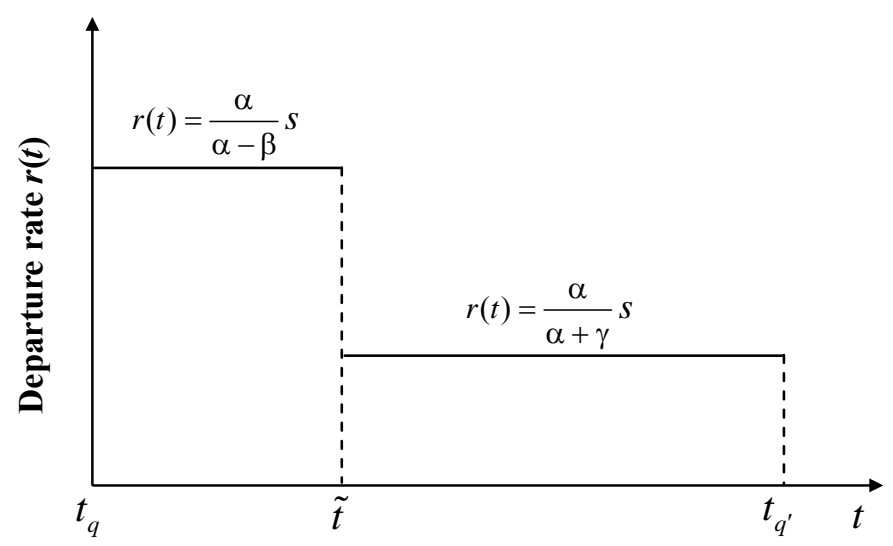

(a)

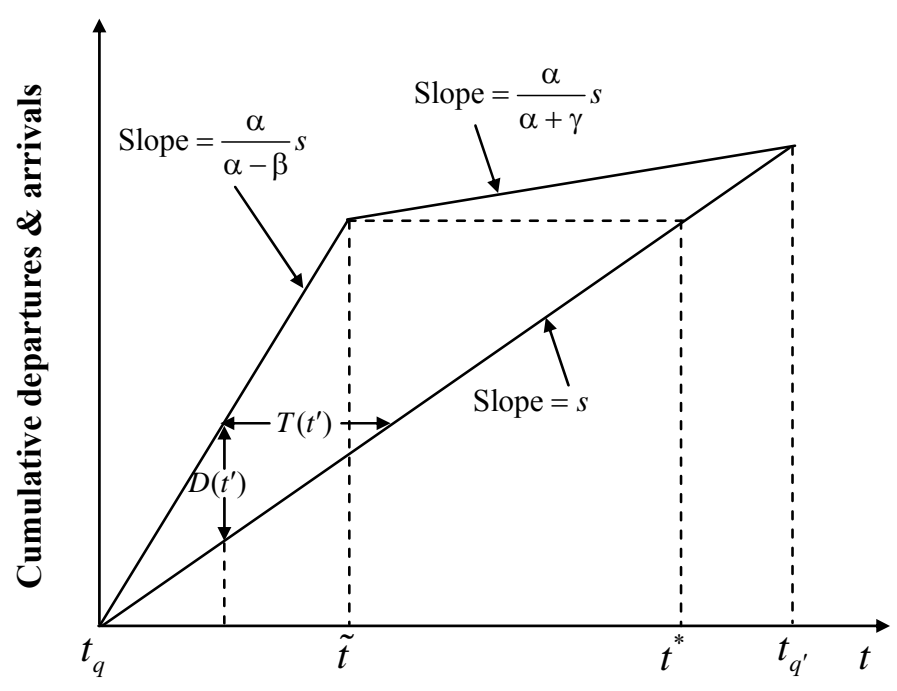

(b)

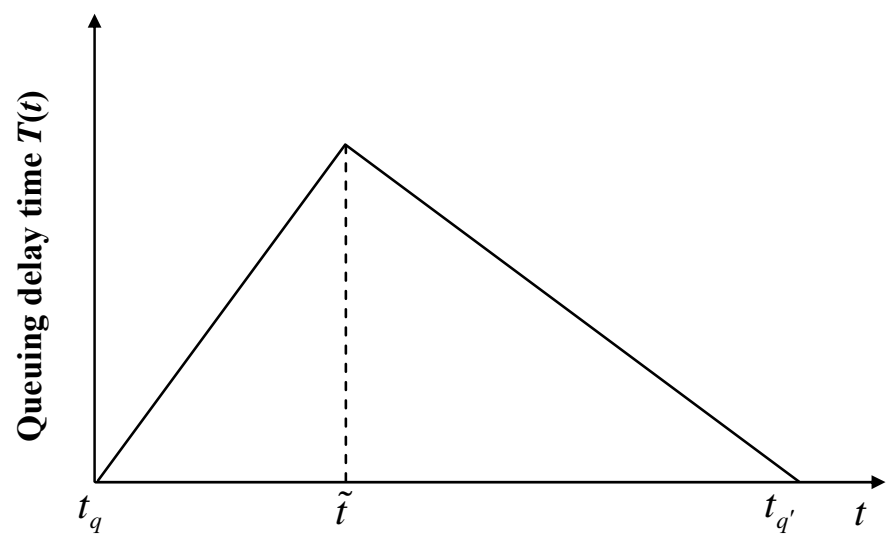

(c)

Fig. 1. Equilibrium solution of Vickrey's bottleneck model: (a) departure rate; (b) cumulative departures and arrivals; (c) queuing delay time. 


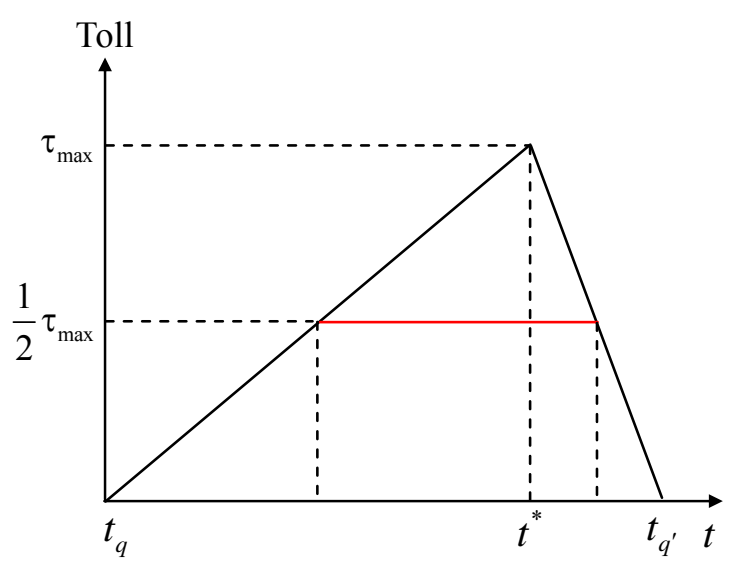

(a)

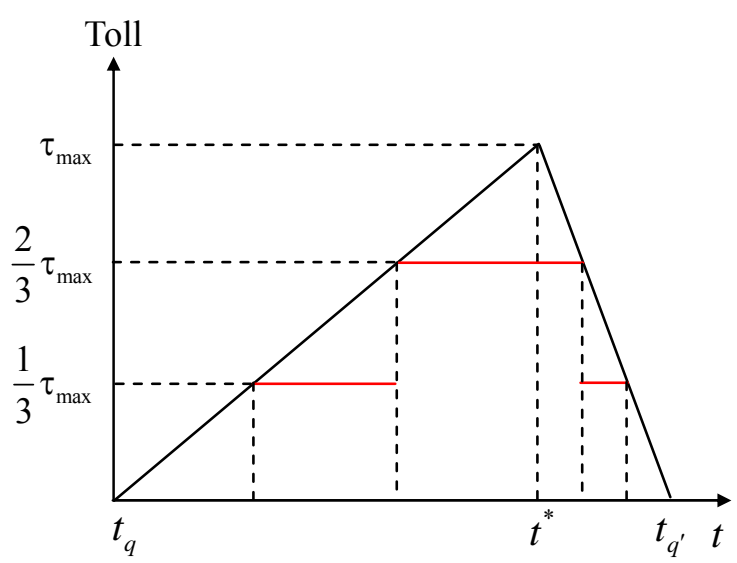

(b)

Fig. 2. Step tolls inscribed in the time-varying toll (Laih, 1994): (a) single-step; (b) two-step. 


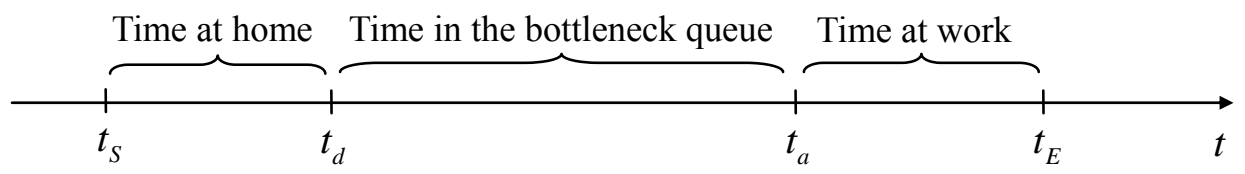

Fig. 3. An example of the morning schedule.

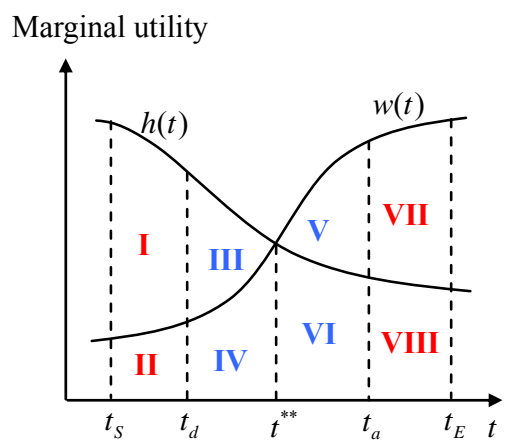

(a)

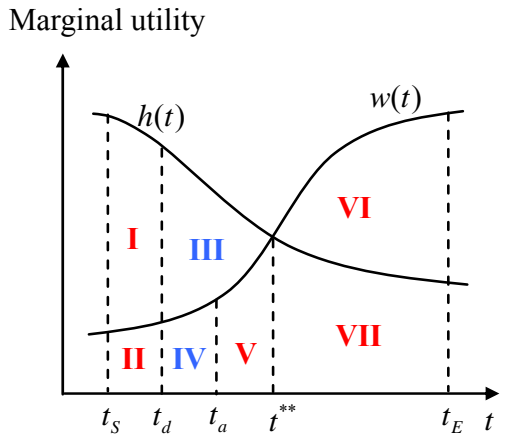

(b)

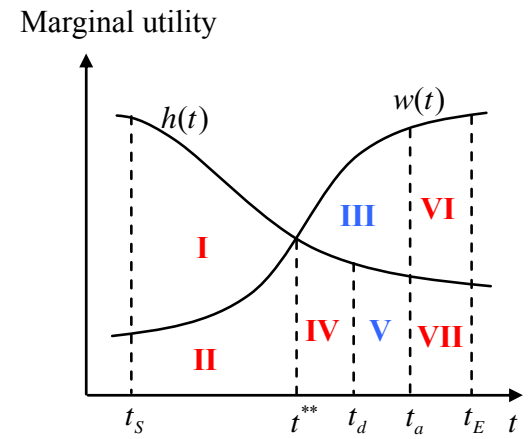

(c)

Fig. 4. Relationships between scheduling utility and scheduling opportunity cost: (a) $t_{d}$ is before $t^{* *}$ and $t_{a}$ is after $t^{* *}$; (b) both $t_{d}$ and $t_{a}$ are before $t^{* *}$; and (c) both $t_{d}$ and $t_{a}$ are after $t^{* *}$. 


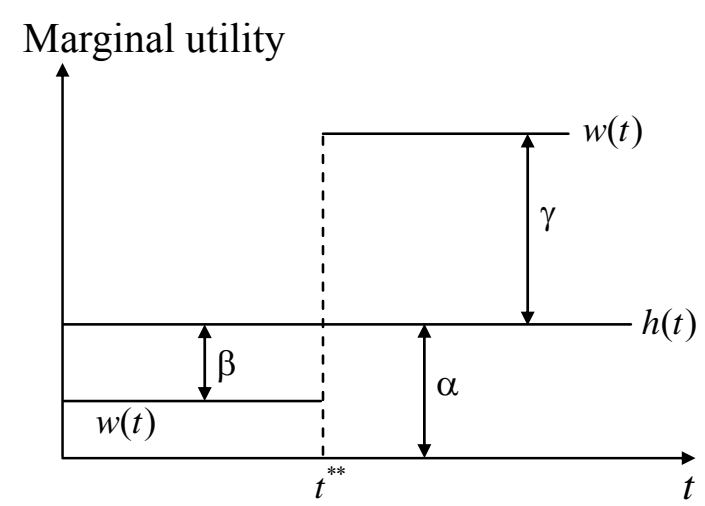

(a)

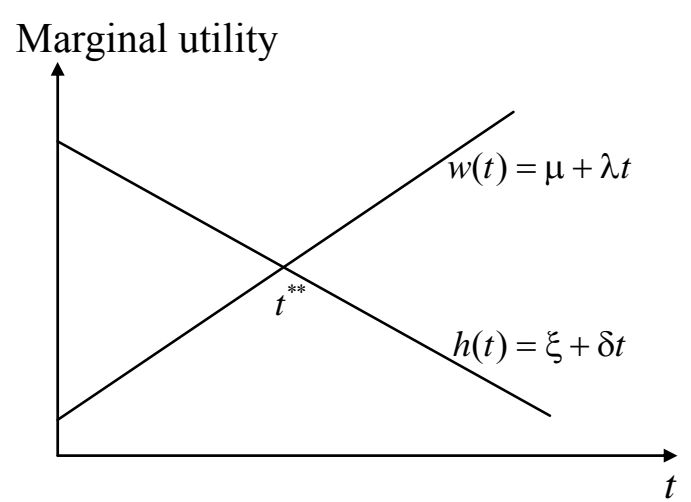

(b)

Fig. 5. Marginal home and work activity utilities: (a) constant; (b) linear. 


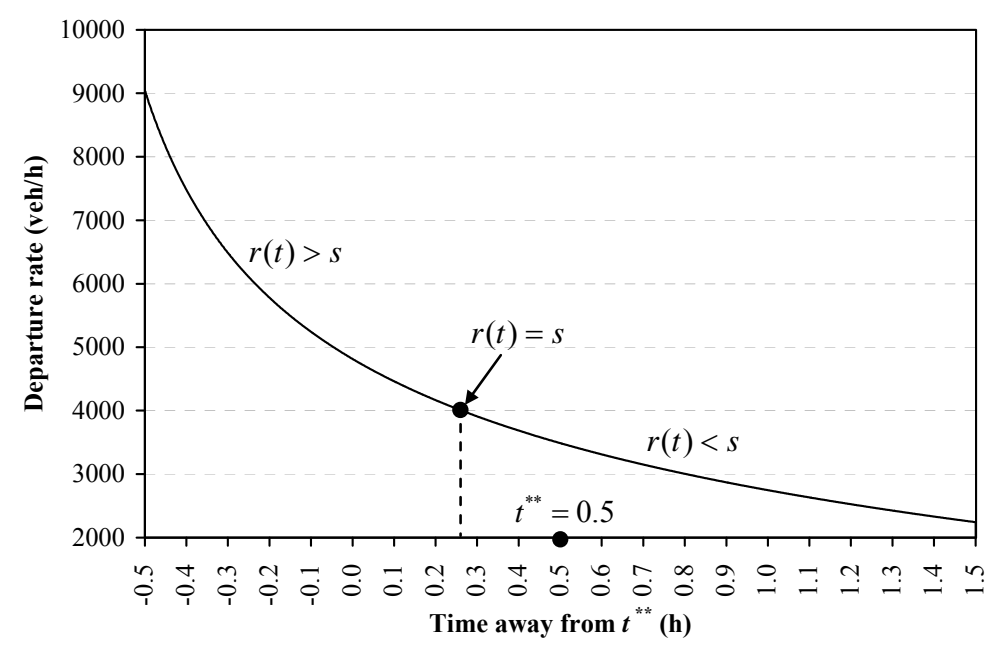

(a)

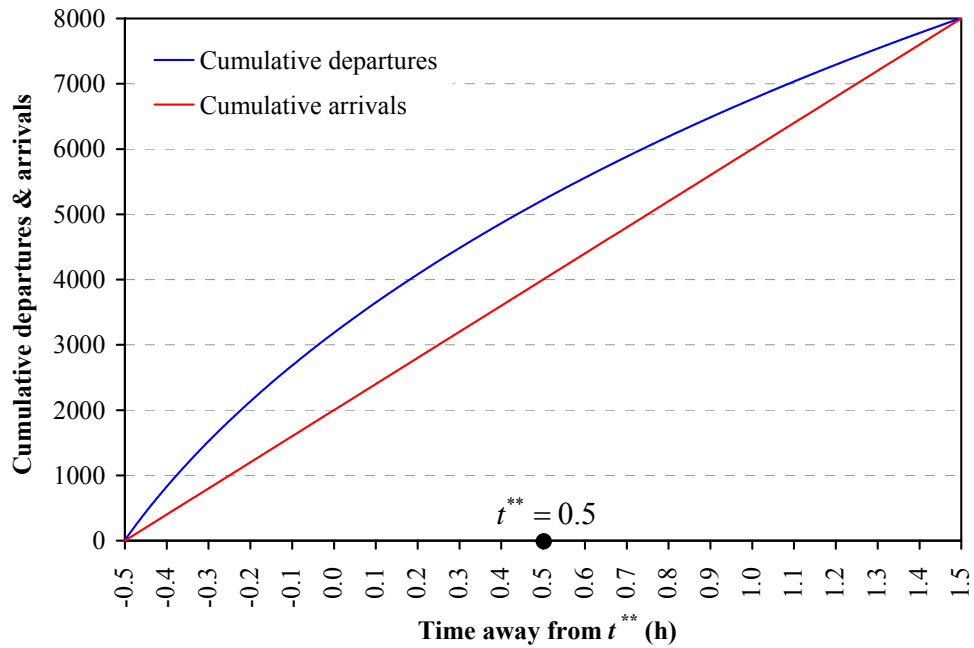

(b)

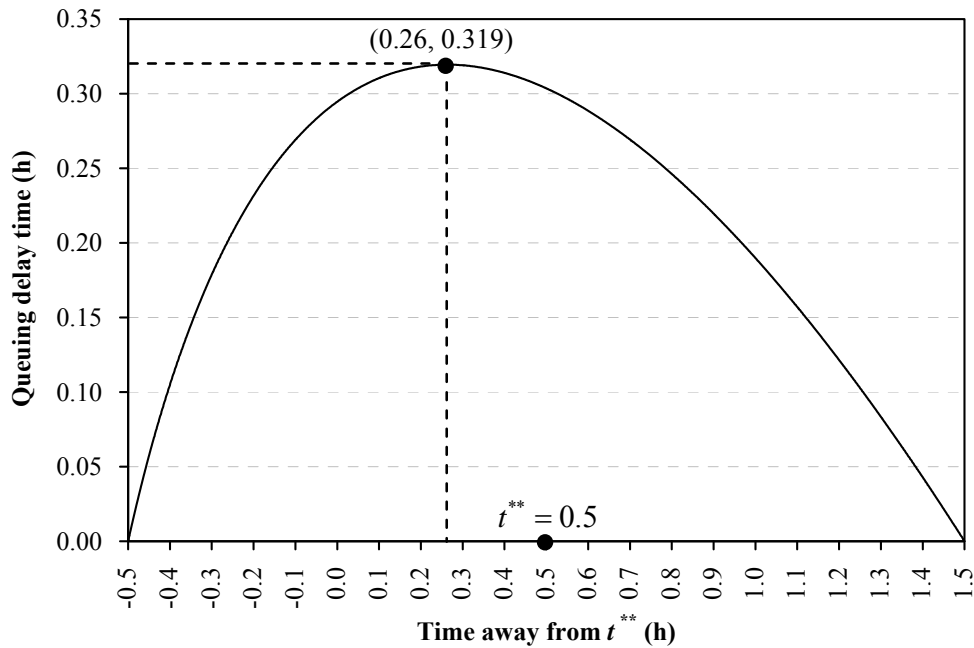

(c)

Fig. 6. No-toll equilibrium during the morning commute: (a) departure rate; (b) cumulative departures and cumulative arrivals; (c) queuing delay time. 


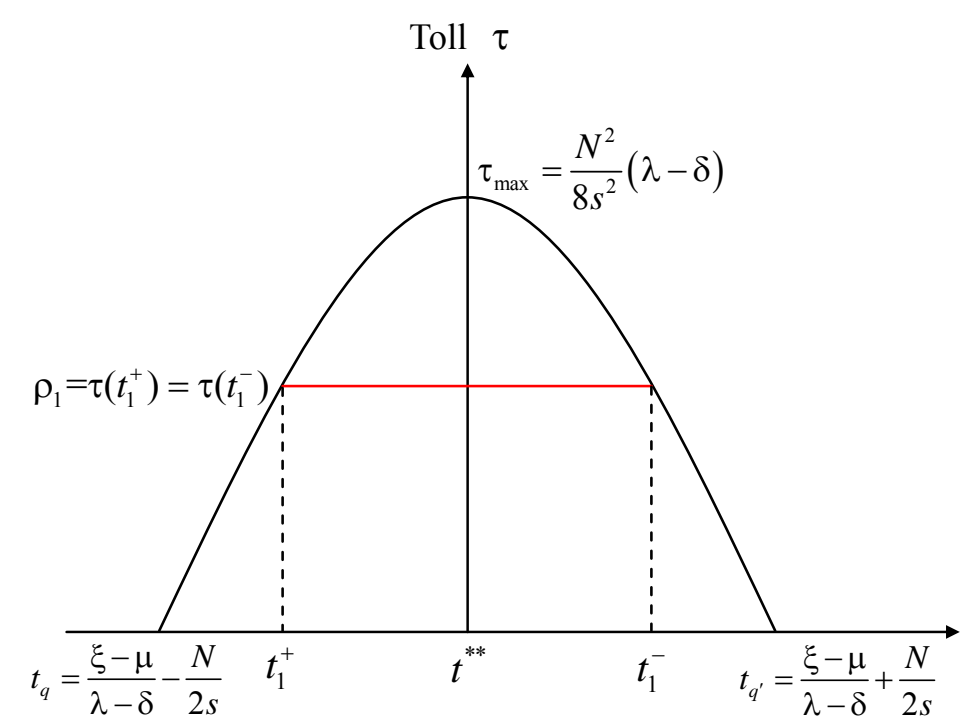

Fig. 7. The single-step toll system inscribed in time-varying toll curve.

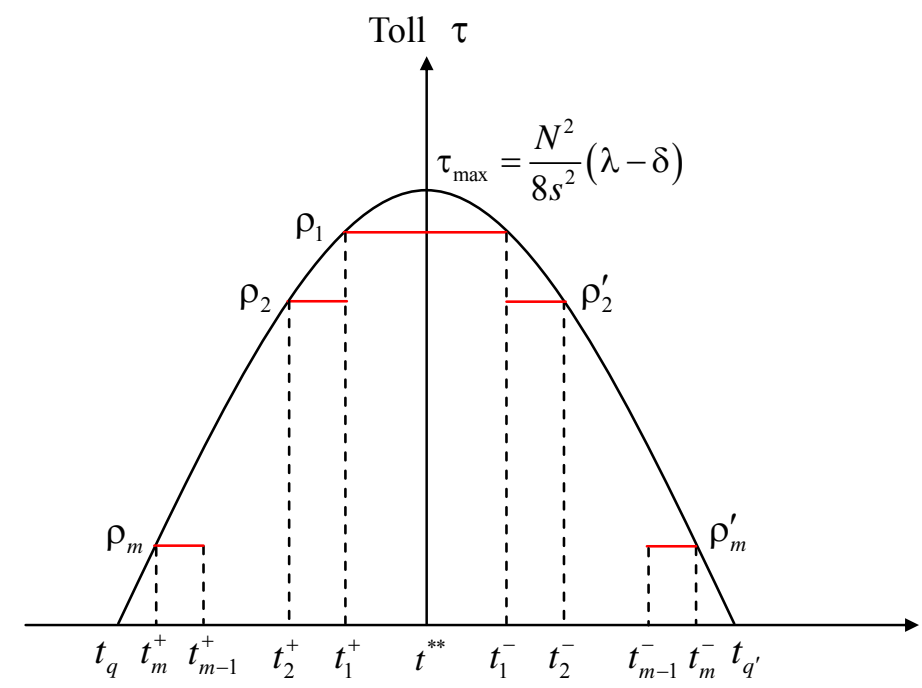

Fig. 8. The $m$-step toll system inscribed in time-varying toll curve. 


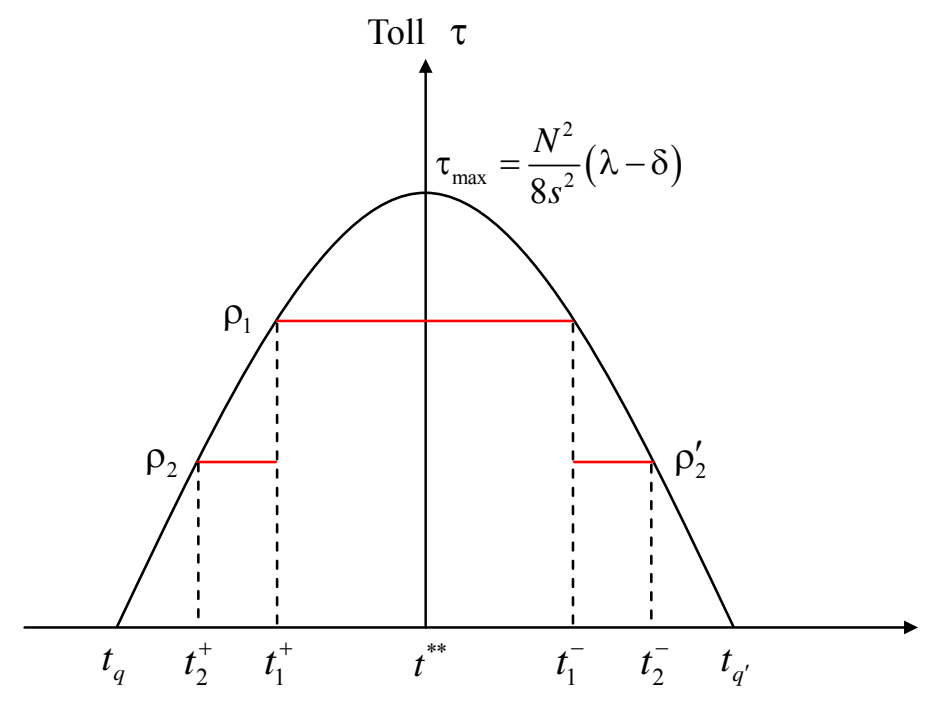

Fig. 9. The two-step toll system.

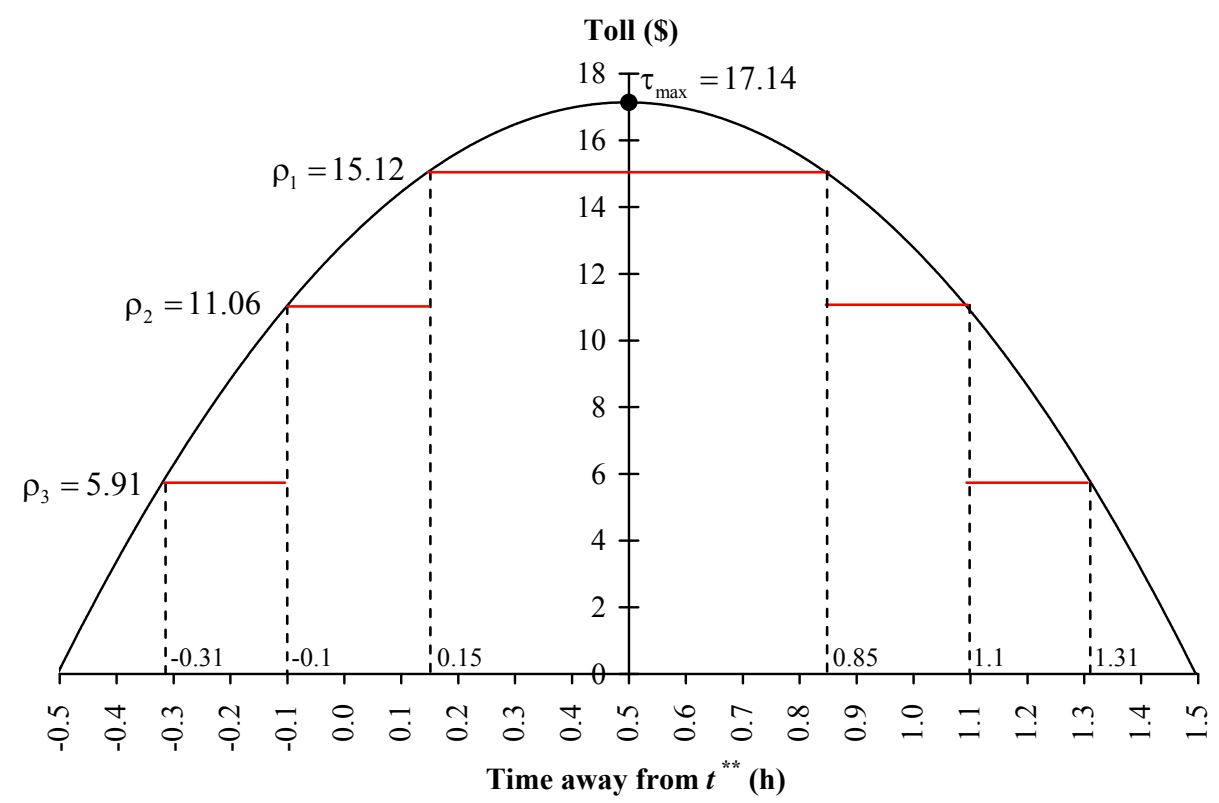

Fig. 10. The three-step toll system. 


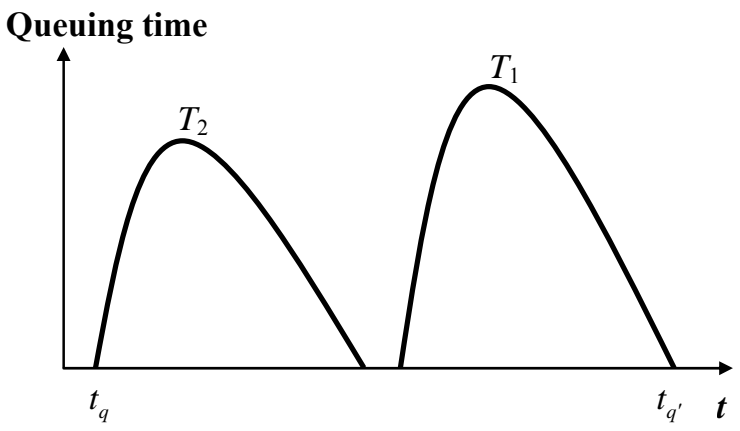

(a)

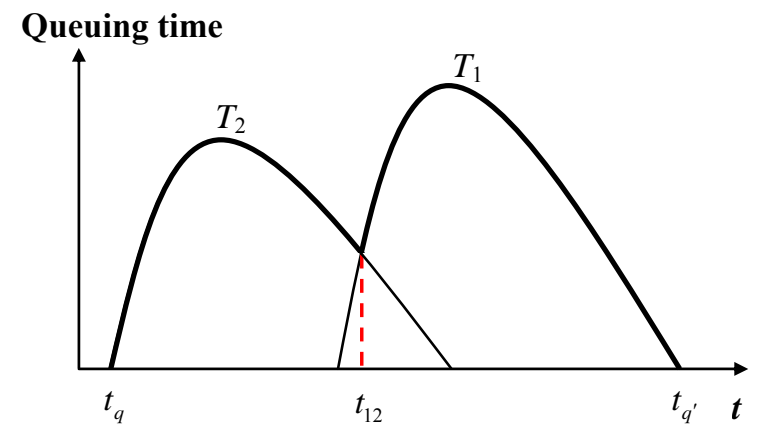

(b)

Fig. 11. Isocost queuing curves for two groups of commuters: (a) two curves isolate; (b) two curves intersect.

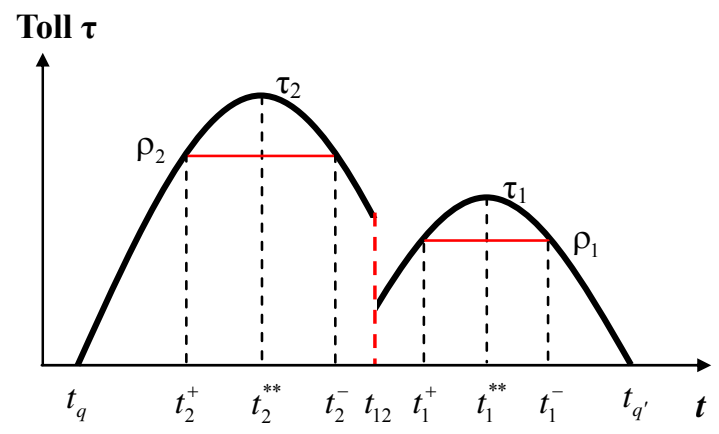

(a)

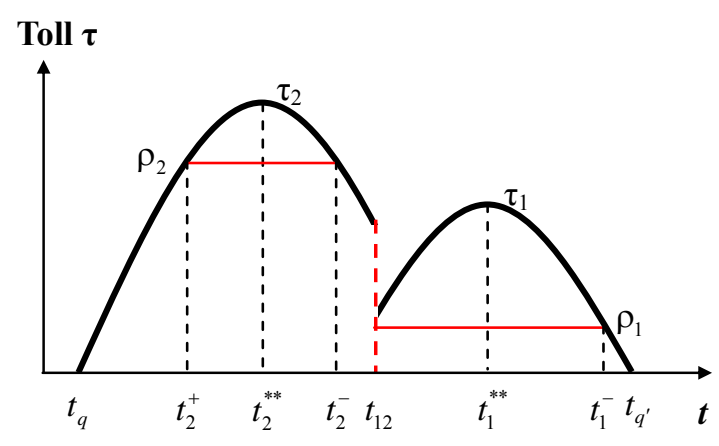

(c)

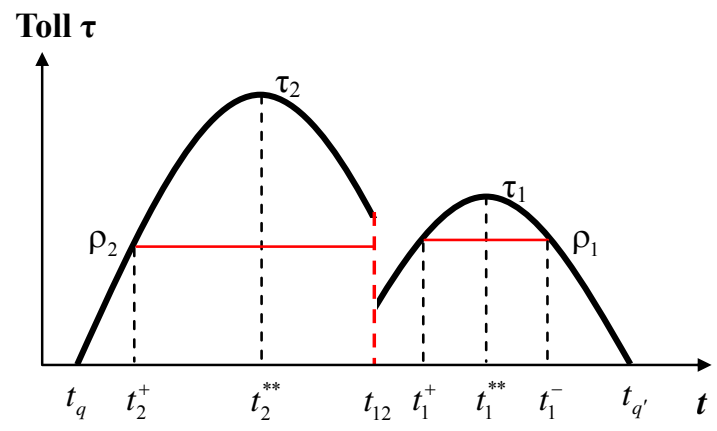

(b)

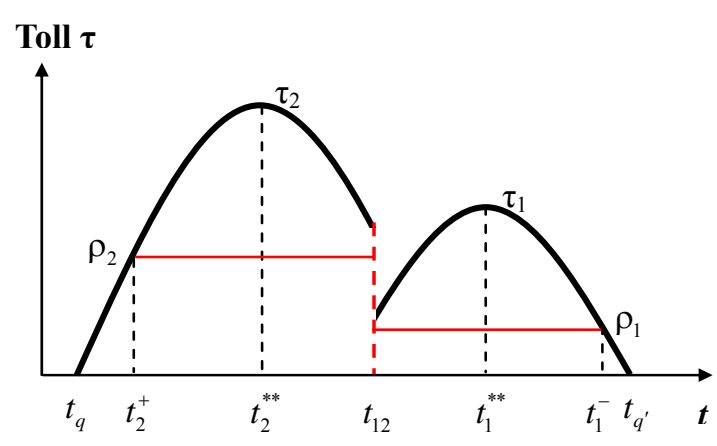

(d)

Fig. 12. Isocost time-varying tolling curves and four scenarios for step toll schemes: (a) Scenario I with $t_{2}^{-} \leq t_{12} \leq t_{1}^{+}$; (b) Scenario II with $t_{12} \leq \min \left(t_{1}^{+}, t_{2}^{-}\right)$; (c) Scenario III with $t_{12} \geq \max \left(t_{1}^{+}, t_{2}^{-}\right) ;(\mathrm{d})$ Scenario IV with $t_{1}^{+} \leq t_{12} \leq t_{2}^{-}$. 


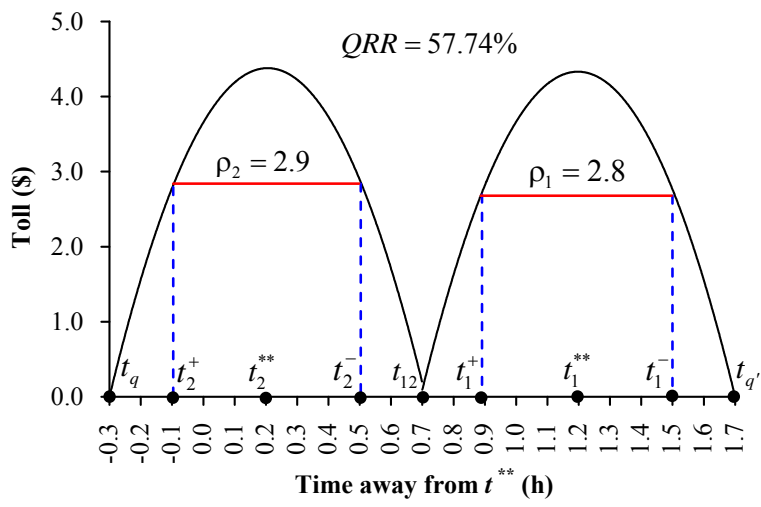

(a)

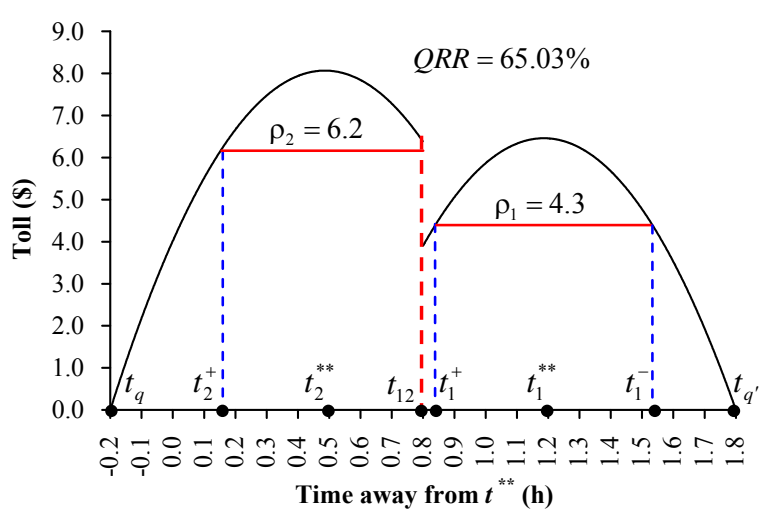

(b)

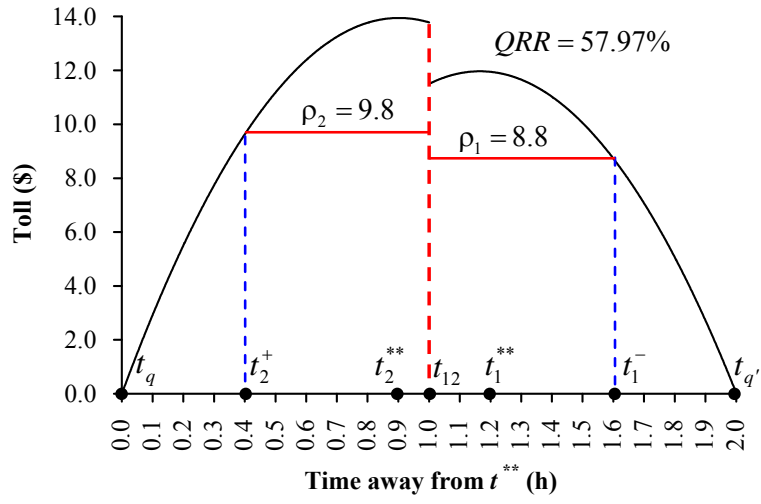

(c)

Fig. 13. Effects of $\mu_{2}$ on optimal step toll scheme for $N_{2}=4000$ : (a) $\mu_{2}=50$; (b) $\mu_{2}=40$; and (c) $\mu_{2}=25$.

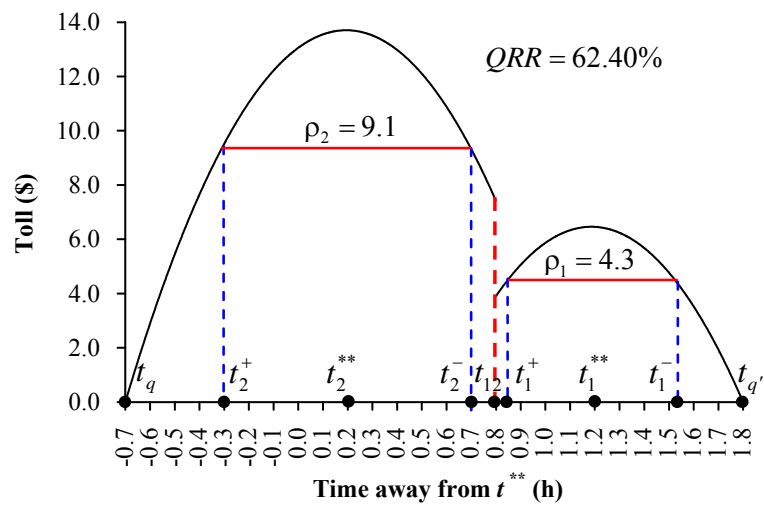

(a)

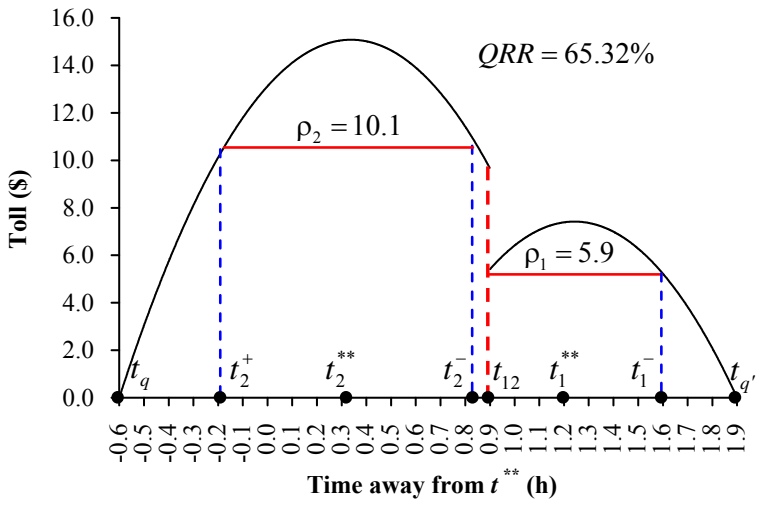

(b)

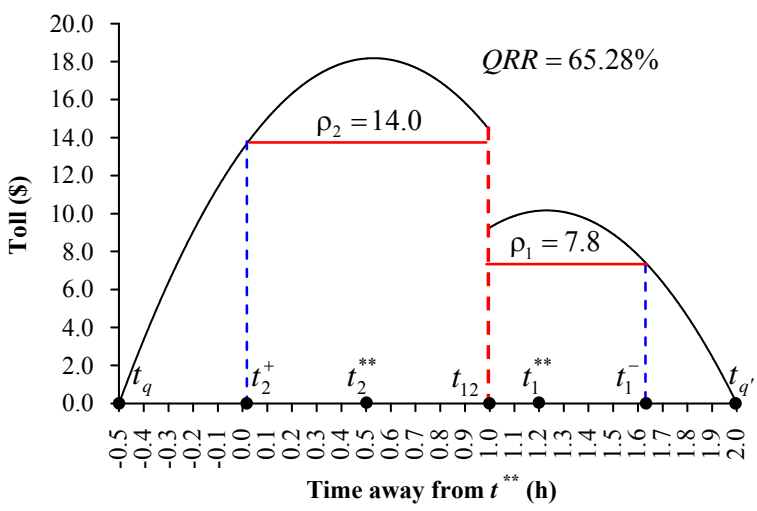

(c)

Fig. 14. Effects of $\mu_{2}$ on optimal step toll scheme for $N_{2}=6000$ : (a) $\mu_{2}=50$; (b) $\mu_{2}=47$; and (c) $\mu_{2}=40$. 


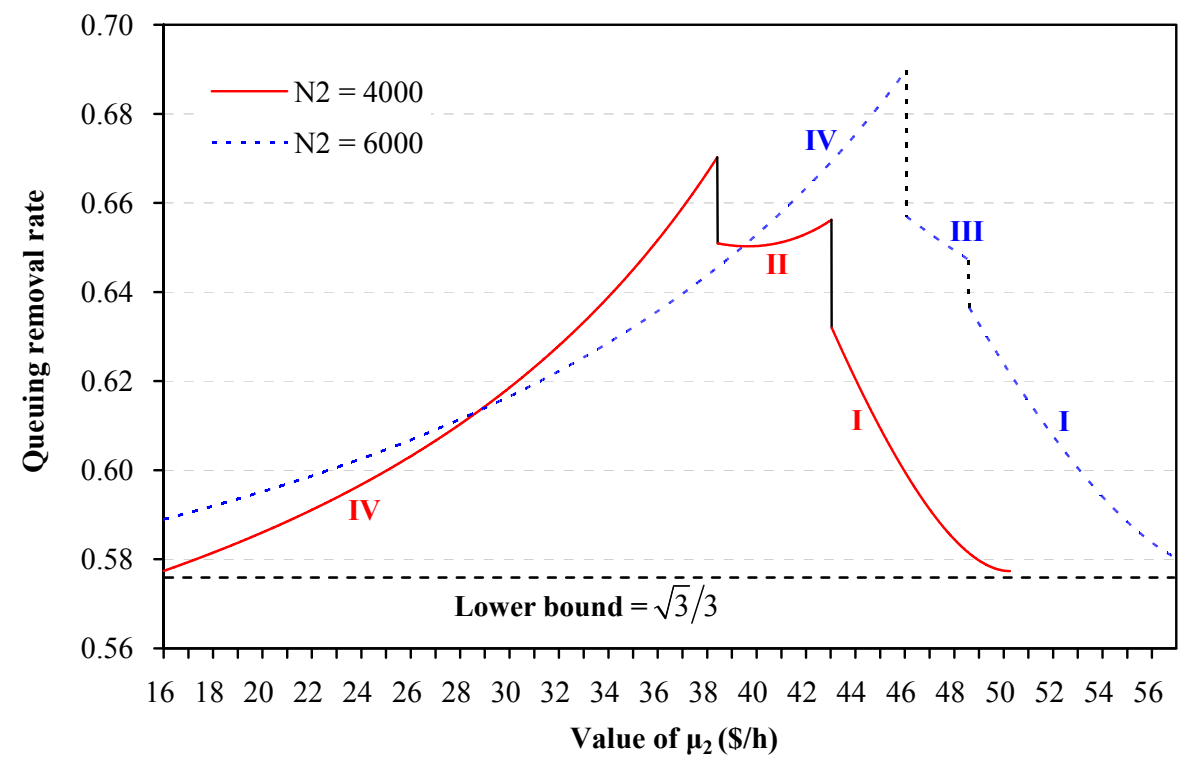

Fig. 15. Changes of step toll models adopted with value of $\mu_{2}$ for different demand levels of group 2 . 